\title{
A QUÍMICA NA AVALIAÇÃO DO IMPACTO À SAÚDE HUMANA DIANTE DA EXPOSIÇÃO AOS PESTICIDAS
}

\author{
Jéssyca Ferreira de Medeiros ${ }^{\mathrm{a}}$, Raphael D’Anna Acayaba ${ }^{\mathrm{a}}$ e Cassiana Carolina Montagner,*,(1) \\ anstituto de Química, Universidade Estadual de Campinas, 13083-970 Campinas - SP, Brasil
}

Recebido em 28/09/2020; aceito em 24/11/2020; publicado na web em 20/01/2021

\begin{abstract}
THE CHEMISTRY IN THE HUMAN HEALTH RISK ASSESSMENT DUE PESTICIDES EXPOSURE. Pesticides are widely used worldwide in urban and rural environments. Since most pesticides are not selective for target species the concern about possible impacts on human health has increased for the workers exposed to these substances (occupational exposure) and for the general population (environmental exposure). Epidemiological studies, in vivo and in vitro have associated several diseases with pesticide exposure, such as cancer, diabetes, Parkinson's, and others. Therefore, chemistry plays an important role in evaluation of external (food and drinking water) and internal (human biomonitoring) exposure to pesticides through of analytical methodologies, for instance, chromatography coupled with mass spectrometry, proving to be an important complement in the evaluation of risks of pesticides in human health.
\end{abstract}

Keywords: pesticides; human health; internal and external exposure; analytical methods.

\section{INTRODUÇÃO}

Pesticidas são substâncias utilizadas no controle de organismos indesejados, incluindo insetos, roedores, fungos e ervas daninhas. ${ }^{1}$ Sua classificação pode ocorrer por diferentes critérios, tais como: origem (orgânica ou inorgânica), estrutura química, toxicidade e finalidade. No entanto, a classificação mais usual considera o tipo de organismos alvo (algicida para algas, acaricida para ácaros, bactericida para bactérias, fungicida para fungos, herbicida para ervas daninhas, inseticidas para insetos, molucida para moluscos, nematicidas para nematoides, rodenticida para roedores, entre outros). ${ }^{2}$ No Brasil é mais frequente a utilização do termo "agrotóxico", definido pela Lei $\mathrm{n}^{\circ} 7.802$, de 11 de julho de 1989 , que evidencia a toxicidade desses produtos ao meio ambiente. ${ }^{3} \mathrm{O}$ Brasil está entre os maiores consumidores de pesticidas no mundo e segundo o Ministério da Agricultura, Pecuária e Abastecimento (MAPA) existem 329 ingredientes ativos ${ }^{4}$ e 2.471 produtos formulados ${ }^{5}$ registrados atualmente.

Os pesticidas são utilizados principalmente para fins agrícolas, entretanto eles também são empregados na saúde pública, em residências e locais públicos. ${ }^{2} \mathrm{Na}$ saúde pública esses compostos são utilizados no controle de doenças transmitidas por vetores, por exemplo, malária e dengue. Em residências e locais públicos os pesticidas são utilizados no controle de plantas indesejadas, paisagismo, parques e jardins para evitar ou suprimir a proliferação de pragas domésticas. Além disso, são empregados em repelentes domésticos, no combate a caspas e piolhos ou no tratamento de animais de estimação, por exemplo, na eliminação de pulgas e carrapatos..$^{6-8}$

A utilização dos pesticidas sintéticos foi iniciada no século $\mathrm{XX}$ e proporcionou muitos benefícios à saúde pública, por exemplo, o suprimento de alimentos se tornou mais abundante e a ocorrência de doenças transmitidas por vetores foi reduzida. Porém, apesar dos benefícios dos pesticidas, seu potencial impacto no meio ambiente e na saúde pública é substancial. ${ }^{9}$ Muller e colaboradores descobriram em 1939 as propriedades inseticidas do 1,1,1-tricloro-2,2-bis(4-clorofenil)-etanol (DDT), um pesticida organoclorado que foi largamente utilizado durante e após a Segunda Guerra Mundial no

*e-mail: ccmonta@unicamp.br combate de vetores de doença, como a malária, e na proteção de culturas. Contudo, em 1962, o livro Primavera Silenciosa de Rachel Carson, mostrou a ameaça que o DDT representava à biodiversidade e deste então, várias outras pesquisas reforçaram sobre o potencial tóxico do DDT que passou a ser proibido em diversos países. ${ }^{10-12}$

Com o banimento ou restrição no uso dos organoclorados em diversos países, novas classes de pesticidas foram desenvolvidas, como os organofosforados, carbamatos, piretróides, neonicotinóides, entre outras. Os organofosforados são caracterizados por uma excelente atividade inseticida e falta de acúmulo biológico e ambiental, porém, esses compostos têm um mecanismo de ação tóxica, baseado na inibição da enzima acetilcolinesterase (AChE), que controla a função nervosa não apenas dos insetos, mas também dos seres humanos e animais. Entre os organofosforados comercializados, tem-se o fosmete, malation, acefato e clorpirifós. Os carbamatos também inibem a $\mathrm{AChE}$, porém formam um complexo menos estável com a colinesterase, permitindo a recuperação da enzima mais rapidamente. Exemplos de pesticidas carbamatos são o carbaril, metomil e carbosulfano. ${ }^{12-14}$ Os piretróides são muito utilizados tanto na agricultura quanto em programas de saúde pública. Por apresentarem uma baixa toxicidade em mamíferos, esses compostos são muito utilizados como inseticidas domésticos. Entre os piretróides utilizados nas residências tem-se a cipermetrina, imiprotrina, transflutrina, praletrina e fenotrina. ${ }^{13,15}$ Os neonicotinóides são utilizados em uma variedade de culturas, eles atuam como agonistas nicotínicos dos receptores de acetilcolina, levando à interrupção dos estímulos nervosos nos insetos e são associados ao declínio global de abelhas. O neonicotinóide imidacloprido é um dos pesticidas mais utilizados no mundo. ${ }^{13,16}$ Atualmente os pesticidas são ainda indispensáveis nas práticas agrícolas e na saúde pública, porém o desenvolvimento de novos compostos que apresentam características menos persistentes e bioacumulativas é de suma importância para oferecer uma maior segurança para os seres vivos e o meio ambiente, que são continuamente expostos a estes compostos.

\section{EXPOSIÇÃO HUMANA AOS PESTICIDAS}

Os pesticidas podem oferecer risco à saúde, tanto para os trabalhadores expostos a essas substâncias quanto para a população em geral. Deste modo, para a liberação de um pesticida, ou seja, 
obtenção do seu registro no mercado, é necessário avaliá-lo quanto ao seu impacto à saúde humana, ao meio ambiente e sua eficácia agrícola. No Brasil, a Agência de Vigilância Sanitária (ANVISA) é o órgão responsável pela avaliação da toxicidade do princípio ativo. ${ }^{2}$ Os pesticidas podem ser encontrados, geralmente em pequenas quantidades, em qualquer lugar no mundo. Dessa forma, os seres humanos estão constantemente sendo expostos a esses compostos e esta exposição ocorre de forma intencional, acidental, ocupacional, ambiental ou pela dieta alimentar. ${ }^{11,12}$

O envenenamento por pesticidas é um problema de saúde pública, principalmente em países em desenvolvimento. No Brasil, os pesticidas são umas das principais causas de intoxicação humana, estando atrás apenas do envenenamento por medicamentos e domissanitários. $\mathrm{O}$ uso intencional de pesticidas ocupa o terceiro lugar no mundo como fonte de tentativa de suicídio devido à sua fácil disponibilidade. Estima-se que esses compostos estejam envolvidos entre 10 a $20 \%$ dos suicídios em todo o mundo. No Brasil, entre 2000 e 2017, houve 48.738 intoxicações e 2.266 óbitos após a tentativa de suicídio pelo uso de pesticidas (agrícolas e domésticos). Os pesticidas também são utilizados na tentativa de aborto, o mesmo estudo mostrou que entre estes anos, 156 pessoas foram envenenadas ao utilizar pesticidas para esta finalidade. ${ }^{17-19}$

A exposição acidental aos pesticidas ocorre principalmente pela via oral. Os casos mais frequentes ocorrem quando os pesticidas são transferidos de seu recipiente rotulado original para um frasco ou recipiente não rotulado. Existem diversos casos em que pessoas são envenenadas por beber pesticidas mantidos em garrafas de refrigerantes ou depois de beber água armazenada em garrafas contaminadas por pesticidas. Os trabalhadores que manipulam pesticidas ou utilizam equipamentos para sua aplicação também podem consumir pesticidas se não lavarem as mãos antes de comer ou fumar. No Brasil houve 55.657 casos relatados de intoxicação acidental por pesticida entre 2000 e 2017. A maioria desses casos ocorreram com indivíduos entre 20-49 anos..$^{2,17,20,21}$ A exposição ocupacional está associada ao ambiente de trabalho, incluindo pessoas envolvidas em indústrias de produção e formulação de pesticidas, agricultores e aplicadores de pesticidas. Na agricultura, a exposição ocorre durante a preparação da calda, aplicação, limpeza dos equipamentos e equipamentos de proteção individual (EPI). Em estudos que avaliaram as características da população rural brasileira foi demonstrado que a maioria dos agricultores entendem que os pesticidas são prejudiciais para a saúde, porém, a utilização de EPIs durante a aplicação não é uma prática comum. Entre 2000 e 2017, 25.038 trabalhadores foram intoxicados com pesticidas no Brasil. ${ }^{17,22-24}$ Os casos de intoxicação intencional, acidental e ocupacional por pesticidas poderiam ser minimizados se essas substâncias fossem armazenadas de modo seguro e se o acesso e manuseio desses produtos fossem mais restritos. Na Figura 1 são apresentadas as intoxicações e óbitos por pesticidas de uso doméstico e agrícola no Brasil obtidos no Sistema Nacional de Informações Tóxico Farmacológicas (SINITOX). ${ }^{17}$

Por outro lado, a exposição ambiental acomete a maioria da população e está relacionada com a presença dos pesticidas em diferentes matrizes ambientais (Figura 2). Após a aplicação, os pesticidas são particionados entre a planta, o solo, a água e a atmosfera. No solo, tais compostos podem sofrer evaporação, fotólise, escoamento superficial, adsorção, degradação química ou biológica, absorção pelas plantas e lixiviação. Sendo o solo, portanto, uma importante via da contaminação do ar e da água. ${ }^{25,26}$ Após a pulverização sobre a área desejada, os pesticidas podem entrar na atmosfera via volatilização direta no momento de sua aplicação ou evaporar posteriormente a partir da superfície do solo ou da vegetação. Uma vez na atmosfera, eles são distribuídos entre as fases gasosa e particulada, dependendo das suas propriedades

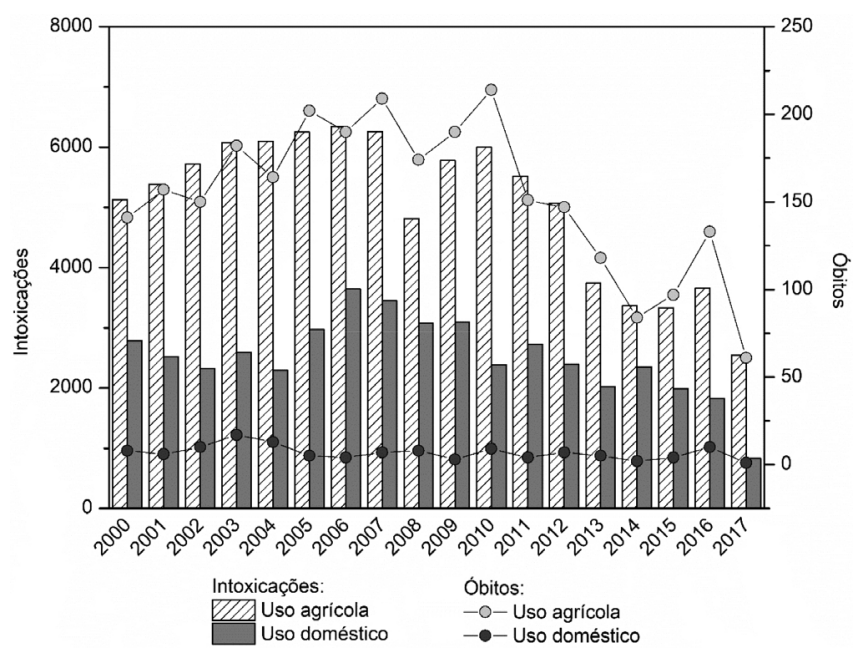

Figura 1. Casos de intoxicação e óbitos por pesticidas de uso doméstico e agrícola no Brasil entre 2000 a 2017 de acordo com os dados do Sistema Nacional de Informações Tóxico Farmacológicas (SINITOX)

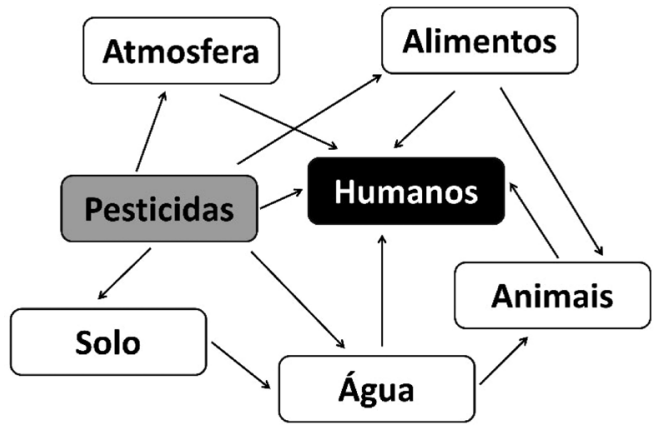

Figura 2. Fontes de exposição ambiental dos seres humanos aos pesticidas

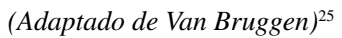

físico-químicas e das condições ambientais. Os pesticidas presentes no ar podem sofrer fotólise, serem transportados pelo vento para áreas distantes do local de aplicação ou atingir a superfície terrestre por deposição úmida ou seca. Assim, eles podem permanecer na atmosfera por dias, semanas ou meses após sua aplicação, possibilitando um risco à saúde pública, bem como aos outros organismos. ${ }^{27,28}$ A contaminação das águas superficiais e subterrâneas por pesticidas tem sido relatada em todo o mundo. Os pesticidas podem atingir águas superficiais e subterrâneas pela ação da chuva e da irrigação que promove a lixiviação, escoamento e drenagem dos pesticidas presentes no solo e nas plantações. Essas substâncias também estão presentes no esgoto, tratado ou não, sendo também uma importante via para a entrada de pesticidas em ambientes aquáticos. Deste modo, a contaminação das águas superficiais e subterrâneas pode resultar em efeitos tóxicos para a saúde humana devido à sua utilização para práticas de lazer ou fontes de água para abastecimento público. ${ }^{29-32}$

No Brasil a presença dos pesticidas no ar e no solo ainda não é legislada. Para matrizes aquáticas como a água potável, água superficial e subterrânea apenas cerca de $8 \%$ do total de pesticidas permitidos para uso são legislados. A Portaria da Consolidação no 5 estabelece a quantidade máxima permitida de 27 pesticidas e alguns dos seus produtos de degradação na água potável. A Resolução CONAMA 357/2005 e 396/2008 tratam da qualidade das águas superficiais e subterrâneas, respectivamente..$^{33-35}$

Os alimentos também são uma importante via da exposição dos seres humanos aos pesticidas. Diversos países estabelecem diretrizes para o monitoramento dos resíduos de pesticidas nos alimentos. 
Neste monitoramento são determinados parâmetros como limites máximos de resíduos (LMR) e ingestão diária aceitável (IDA) que visam proteger a saúde humana. O LMR é definido como a quantidade máxima (expressa em $\mathrm{mg} \mathrm{kg}^{-1}$ ) de resíduo do pesticida que é legalmente permitida em alimentos. A IDA é um parâmetro de segurança definido como a quantidade estimada do pesticida nos alimentos que pode ser ingerida diariamente durante toda a vida, sem riscos consideráveis à saúde do consumidor. ${ }^{36-38}$ No Brasil, os níveis de resíduos de pesticidas nos alimentos de origem vegetal são avaliados pela ANVISA por meio do Programa de Análise de Resíduos de Agrotóxicos em Alimentos (PARA) ${ }^{39}$ e também através do Plano Nacional de Controle de Resíduos e Contaminantes (PNCRC) ${ }^{40}$ vinculado ao MAPA. Além de realizar o monitoramento em vegetais, o PNCRC também monitora os alimentos de origem animal. Em 2018, 5.654 amostras de alimentos foram analisadas pelos programas da ANVISA e MAPA e, desse total, 1.106 foram consideradas em não conformidade. ${ }^{39,40}$ No entanto, ainda que a concentração dos pesticidas nos alimentos esteja abaixo dos LMRs, o risco à saúde da população não pode ser descartado, pois são necessárias abordagens mais complexas para a avaliação do risco para a saúde humana. Nessa avaliação deve-se levar em consideração a exposição cumulativa crônica e agregada a estes compostos..$^{39,41}$

As vias mais comuns de absorção de pesticidas no corpo humano são a dérmica, oral e respiratória. Após a entrada, eles são distribuídos, em sua maioria, através da corrente sanguínea e podem ser excretados pela urina, pele e ar exalado. ${ }^{2,20}$ A via dérmica é uma das rotas mais comuns pelas quais os aplicadores de pesticidas são expostos. A absorção dérmica pode ocorrer como resultado de respingos e derramamentos durante a mistura, carregamento ou descarte dos pesticidas. $\mathrm{O}$ risco da absorção dérmica depende da quantidade do pesticida, da sua toxicidade para a pele, duração da exposição, formulação do pesticida e do uso de equipamentos de proteção individual. Os pesticidas em pó ou granulares têm uma menor absorção na pele em relação às formulações líquidas. Embora a via dérmica, em geral, seja considerada a principal via de exposição ocupacional a pesticidas, a inalação não deve ser negligenciada. A inalação de partículas ou vapores pode ocorrer durante o manuseio e a aplicação dos pesticidas. Esses compostos são rapidamente absorvidos pela via respiratória, podendo causar danos ao nariz, garganta e tecidos pulmonares. O potencial de envenenamento é maior quando a solução de pulverização forma vapores e partículas extremamente finas. Essa exposição pode ser ainda mais significativa se os pesticidas são aplicados em espaços confinados, por exemplo, em áreas de armazenamento não ventiladas ou em estufas. ${ }^{42-47} \mathrm{Na}$ agricultura familiar brasileira geralmente são utilizados equipamentos de baixa tecnologia para a aplicação de pesticidas, como o pulverizador costal, além disso, muitos aplicadores não utilizam ou fazem o uso inadequado dos EPIs podendo ser expostos dermicamente durante a mistura ou inalarem a névoa durante a aplicação..$^{21,48,49}$

$\mathrm{O}$ envenenamento mais grave pode ocorrer quando o pesticida é introduzido oralmente. Esse tipo de exposição ocorre por razões acidentais ou intencionais. Após serem ingeridos, os pesticidas podem ser absorvidos ao longo do trato gastrointestinal e atingir a corrente sanguínea, podendo se distribuir por todo o organismo. ${ }^{2,43}$

\section{EFEITOS DOS PESTICIDAS NA SAÚDE HUMANA}

Nos últimos anos, os efeitos do uso de pesticidas na saúde humana têm sido muito estudados. Estudos observacionais em trabalhadores expostos aos pesticidas em conjunto com estudos in vivo e in vitro, demonstraram que diversos pesticidas são associados a efeitos prejudiciais à saúde. Entretanto, fazer uma associação direta não é simples, pois o efeito pode ser associado a diferentes fatores, como pré-disposição genética, tabagismo, envelhecimento, entre outros. A fim de buscar cenários mais críticos e, portanto, onde as evidências podem ser mais facilmente elucidadas, a maioria dos estudos na literatura são voltados para a exposição ocupacional. Assim, a exposição ambiental, que embora engloba a maior parte da população, ainda em concentrações menores e majoritariamente pela exposição crônica, não é muito avaliada. . $^{43,47,50}$

Os efeitos à saúde devido à exposição não dependem apenas da toxicidade dos ingredientes ativos, mas também da quantidade do produto absorvido e do tempo de exposição. ${ }^{2}$ Esses efeitos podem ser agudos (de manifestação rápida) ou crônicos (que aparecem após exposições repetidas a pequenas quantidades dos pesticidas por um longo período). Os efeitos relacionados à exposição aguda podem ter gravidade baixa (ex.: dor de cabeça), moderada (ex.: diarreia), alta (ex.: edema pulmonar) ou serem fatais. Irritação na pele, desidratação, alergias, ardência do nariz, tosse, coriza, dor no peito, dificuldade de respirar, irritação da boca e garganta, dor de estômago, náuseas, vômitos, diarreia, dor de cabeça, transpiração anormal, fraqueza, câimbras e tremores são alguns dos efeitos agudos associados à exposição aos pesticidas. ${ }^{51,52}$

Diversas evidências relacionaram a exposição aos pesticidas e a taxa elevada de doenças crônicas, tais como, diabetes, ${ }^{53}$ obesidade, ${ }^{54}$ diferentes tipos de câncer, ${ }^{22}$ distúrbios neurodegenerativos (por exemplo, doença de Parkinson, ${ }^{55}$ Alzheimer ${ }^{56}$ e Esclerose Lateral Amiotrófica), ${ }^{57}$ defeitos congênitos, ${ }^{58}$ doenças reprodutivas, ${ }^{59}$ respiratórias, ${ }^{60}$ cardiovasculares,${ }^{61}$ entre outros. ${ }^{22,62}$ Os pesticidas modulam várias atividades celulares e subcelulares que podem levar a alterações genéticas causando várias doenças ou morte celular. Estudos experimentais demostraram que os pesticidas podem ocasionar danos no DNA, mutação genética, aberrações cromossômicas e modificações epigenéticas. Essas alterações estão ligadas ao desenvolvimento de cânceres e à indução de alterações epigenéticas que podem ser transmitidas às gerações subsequentes ou podem servir como base para doenças desenvolvidas posteriormente na vida. ${ }^{26,62,63}$

Os pesticidas podem causar desregulação endócrina através da interrupção da atividade, tempo de liberação ou podem imitar hormônios do sistema endócrino que resultam na redução da fertilidade, irregularidades das glândulas da tireoide e comprometimento da função imunológica. ${ }^{26,62} \mathrm{~A}$ atrazina é considerada um desregulador endócrino. Em Illinois, EUA, em um estudo epidemiológico preliminar com mulheres que moravam na área rural onde a atrazina era usada extensivamente, foi observado que elas apresentaram irregularidades no ciclo menstrual com fases foliculares mais longas e níveis reduzidos de metabólitos de estradiol e progesterona. ${ }^{64}$

Os pesticidas também foram associados com a doença de Parkinson através da inibição da função mitocondrial. Os mecanismos específicos pelos quais esses compostos afetam os neurônios ainda são desconhecidos e apesar das evidências epidemiológicas e experimentais, ainda é difícil concluir que a exposição aos pesticidas possa causar a doença de Parkinson. ${ }^{22,47,65}$ Estudos associaram que pessoas expostas ocupacionalmente aos pesticidas tem um risco aumentado de desenvolvimento da doença de Alzheimer, de transtornos mentais, como depressão e ansiedade, e tentativas de suicídio. ${ }^{66-68}$

Através de estudos in vitro e in vivo foi observado que os pesticidas, tais como organoclorados e organofosforados, podem alterar o metabolismo da glicose de modo a ocasionar o desenvolvimento da diabetes. Como as evidências da ligação entre diabetes e uso de pesticidas são relativamente novas, ainda são necessárias pesquisas para descobrir mais detalhes sobre essa associação., 2,69 Por outro lado, a ligação entre a exposição aos pesticidas e o desenvolvimento de câncer tem sido relatada por muitos estudos. Por meio de evidências 
crescentes fornecidas por estudos epidemiológicos foram relatadas associações entre pesticidas e o desenvolvimento de diversos tipos de cânceres em crianças e adultos, tais como, câncer de mama, ${ }^{70}$ próstata, ${ }^{71}$ pulmão,${ }^{72}$ linfoma não-Hodgkin, ${ }^{73}$ leucemia, ${ }^{74}$ entre outros.

Os pesticidas também foram associados com a indução de sinais inflamatórios na célula e a geração de espécies reativas de oxigênio (EROs). As EROs podem levar ao acúmulo de agregados de proteínas desdobradas através da deformação do sistema ubiquitina-proteassoma. A desregulação desse sistema tem sido implicada na patogênese de várias doenças crônicas, principalmente neurodegeneração e cânceres. O pesticida organoclorado dieldrin foi associado à diminuição da atividade da proteassoma juntamente com um aumento da ocorrência de apoptose em células neuronais. ${ }^{62,75,76}$ Os pesticidas também podem induzir o estresse do retículo endoplasmático, que está associado com o desenvolvimento de várias doenças, tais como diabetes, ${ }^{77}$ Parkinson e Alzheimer. ${ }^{78}$ Quando os mecanismos de defesa antioxidante são sobrecarregados, o estresse oxidativo pode causar danos no DNA, nas proteínas e lipídios das células, levando a alterações cromossômicas, mutação genética e/ou crescimento celular que, por fim, podem resultar em cânceres. ${ }^{62,79,80}$ A Figura 3 apresenta de forma simplificada algumas alterações que os pesticidas podem ocasionar na célula.

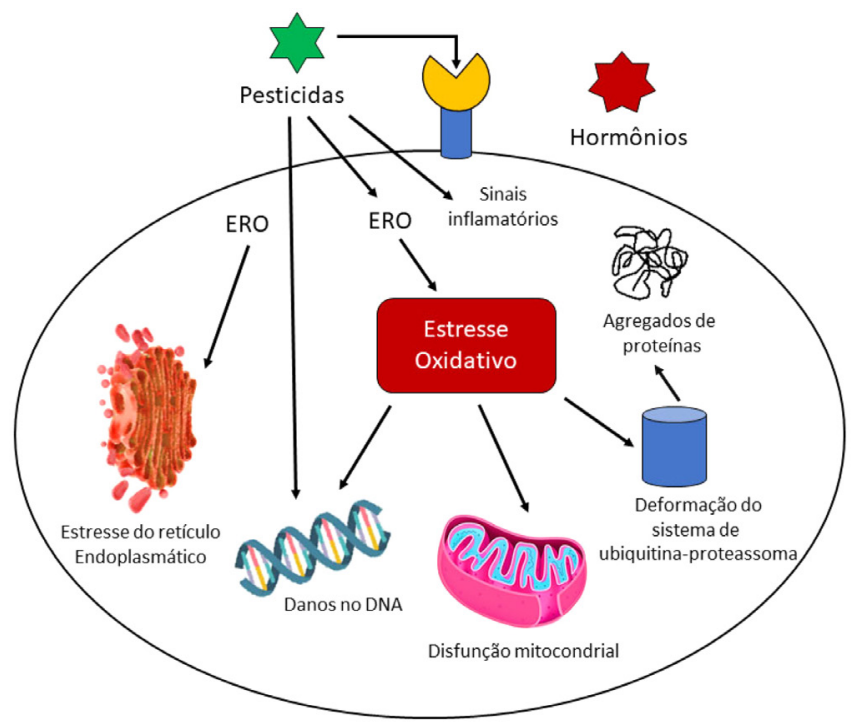

Figura 3. Modelo simplificado das alterações que os pesticidas podem ocasionar na célula (Adaptado de Sabarwal, Kumar e Singh) ${ }^{22}$

Em função da variedade de ingredientes ativos utilizados, a população, em geral, é exposta a uma mistura dessas substâncias. Deste modo, pesticidas que compõe uma mistura podem interagir entre si e o efeito dessa interação pode ser sinérgico ou antagônico. Por exemplo, na exposição simultânea de organofosforados geralmente é observado um efeito somado dos compostos individuais na inibição da atividade da enzima acetilcolinesterase (AChE). O entendimento dos efeitos devido às misturas de pesticidas na saúde humana ainda é limitado. ${ }^{81-83}$ Exames laboratoriais podem ser realizados para estimar a exposição aos pesticidas organofosforados e carbamatos. Estes pesticidas inibem a atividade das colinesterases, principalmente da AChE, aumentando o nível da acetilcolina nas sinapses químicas. O acúmulo de acetilcolina promove a toxicidade colinérgica, que pode ocasionar sintomas muscarínicos, nicotínicos e neurológicos. ${ }^{84-88}$ Os organofosforados e outros agentes inibidores da colinesterase, como os carbamatos, têm sido associados a transtornos psiquiátricos e comportamentos suicidas. ${ }^{67,89}$

\section{MÉTODOS ANALÍTICOS EMPREGADOS NA DETERMI- NAÇÃO DE PESTICIDAS EM ÁGUA, EM ALIMENTOS E EM MATRIZES BIOLÓGICAS}

A determinação dos pesticidas na água, nos alimentos e em matrizes biológicas geralmente envolve uma etapa de pré-tratamento da amostra, seguida por um processo de extração e limpeza e, finalmente, um método de separação e detecção. ${ }^{90-92} \mathrm{Na}$ Figura 4 são apresentados os principais métodos analíticos empregados na avaliação de pesticidas na água, nos alimentos e em matrizes biológicas.

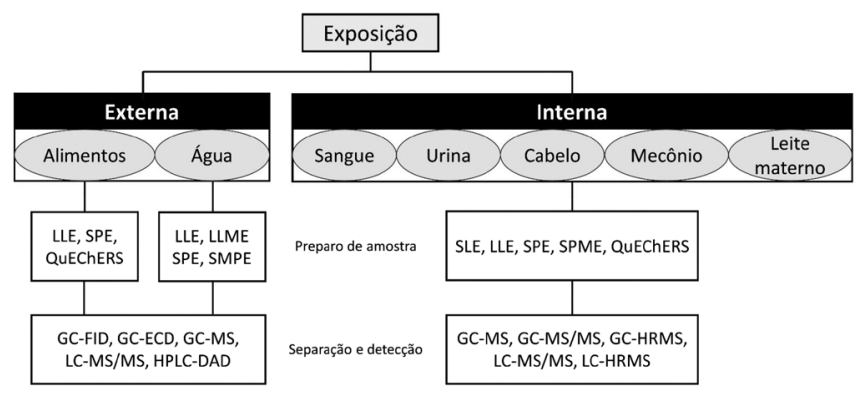

Figura 4. Principais técnicas analíticas empregadas no preparo de amostras e na separação e detecção de pesticidas em água, nos alimentos e em matrizes biológicas

\section{ÁGUA}

A maioria dos pesticidas estão presentes no ambiente aquático em concentrações na ordem de picograma a nanograma por litro e, portanto, para sua determinação são necessários métodos analíticos com baixos limites de detecção. ${ }^{92,93}$ As técnicas mais empregadas na extração, purificação e pré-concentração dos pesticidas na água são a extração líquido-líquido (LLE, do inglês liquid-liquid extraction), ${ }^{94,95}$ microextração líquido-líquido (LLME, do inglês liquid-liquid microextraction), ${ }^{96-98}$ extração em fase sólida (SPE, do inglês solid-phase extraction) nas configurações offline ${ }^{99-101} \mathrm{e}$ online ${ }^{102-104}$ e microextração em fase sólida (SPME, do inglês solidphase microextraction). ${ }^{93,105,106} \mathrm{Na}$ separação e determinação dos pesticidas em matrizes aquáticas as técnicas mais empregadas são a cromatografia gasosa (GC, do inglês gas chromatography) ou líquida (LC, do inglês liquid chromatography) acoplada à espectrometria de massas (MS, do inglês mass spectrometry). A técnica de GC-MS é mais utilizada na determinação de compostos apolares, voláteis e termicamente estáveis e a LC-MS/MS tem sido mais empregada na análise de compostos orgânicos polares e semipolares. ${ }^{93,107,108}$ A Tabela 1 apresenta algumas técnicas e parâmetros analíticos empregados na determinação de pesticidas em amostras de água.

Resíduos de pesticidas têm sido detectados em mananciais e água de abastecimento público em todo o mundo, incluindo no Brasil. Montagner e colaboradores ${ }^{101}$ desenvolveram um método para avaliar a ocorrência de 12 pesticidas (atrazina, carbendazim, clorpirifós, profenofós, difenoconazol, epoxiconazol, tebuconazol, azoxistrobina, picoxistrobina, piraclostrobina, trifloxistrobina e fipronil) em águas superficiais e de abastecimento público do estado de São Paulo, Brasil. Os analitos foram extraídos por SPE com os cartuchos Oasis HLB, Strata SAX, C18 Envi-18 e Envi Carb. O sorvente Oasis HLB com os solventes de eluição metanol seguido de acetonitrila proporcionaram uma melhor recuperação da maioria dos pesticidas (> 70\%). Os limites de detecção (LD) e quantificação (LQ) do método foram 1-50 e 2-150 $\mathrm{ng} \mathrm{L}^{-1}$, respectivamente. Nas amostras de água superficial foram detectados 9 pesticidas (atrazina, carbendazim, difenoconazol, epoxiconazol, tebuconazol, azoxistrobina, picoxistrobina, piraclostrobina e trifloxistrobina) em 
Tabela 1. Metodologias analíticas para determinação de pesticidas em água de abastecimento público (cont.)

\begin{tabular}{|c|c|c|c|c|c|}
\hline Pesticidas & $\begin{array}{l}\text { Preparo de } \\
\text { amostra }\end{array}$ & $\begin{array}{c}\text { Análise } \\
\text { instrumental }\end{array}$ & LD & LQ & Referência \\
\hline $\begin{array}{l}\text { Metiocarbe, oxadiazon, imidacloprida, tiacloprido, tiametoxame, } \\
\text { clotianidina, acetamiprido, triallate }\end{array}$ & SPE & LC-MS/MS & $0,1-1,4 \mathrm{ng} \mathrm{L}^{-1}$ & $0,3-4,8 \mathrm{ng} \mathrm{L}^{-1}$ & 125 \\
\hline $\begin{array}{l}\text { Clopiralide, diuron, florasulam, CGA 282149, CGA 322704, } \\
\text { CGA 353968, CGA 355190, NOA 404617, NOA 407475, 2,4- } \\
\text { D, 2,4,5-T, 2,6- ácido difluorobenzoico, bentazone, dicamba, } \\
\text { diclorprop, dimetomorfo, flumetsulame, imidacloprida, linuron, } \\
\text { MCPA, mecoprop, penconazol, tiametoxame }\end{array}$ & SPE online & LC-MS/MS & $1,2-18,0 \mathrm{ng} \mathrm{L}^{-1}$ & & 126 \\
\hline $\begin{array}{l}\text { ametrina, atrazina, cianazina, prometrina, propazina, simazina, } \\
\text { simetrin, terbutilazina, terbutrina }\end{array}$ & SPE & HPLC-DAD & $0,010-0,023 \mu \mathrm{g} \mathrm{L}^{-1}$ & & 127 \\
\hline
\end{tabular}

LD: limite de detecção; LQ: limite de quantificação; SPE: extração em fase sólida (do inglês, solid-phase extraction); LLE: líquido-líquido (do inglês, liquidliquid extraction); DLLE: microextraçao líquido-líquido dispersiva (do inglês, dispersive liquid-liquid microextraction); SPME: microextração em fase sólida (do inglês, solid-phase microextraction); DPX: extração em ponteiras descartáveis (do inglês, disposable pipette extraction); GC: cromatografia gasosa (do inglês, gas chromatography); LC: cromatografia líquida (do inglês, liquid chromatography); UHPLC: cromatografia líquida de ultra eficiência (do inglês, ultra high performance liquid chromatography); HPLC: cromatografia líquida de alta eficiência (do inglês, ultra high performance liquid chromatography); MS: espectrometria de massas (do inglês, mass spectrometry); MS/MS: espectrometria de massas sequencial (do inglês, tandem mass spectrometry); DAD: detector de arranjo de diodos (do inglês; diode array detector); ECD: detector de captura de elétrons (do inglês, electron capture detector)

concentrações entre 3 e 293 ng L-1. Na água tratada, o tebuconazol, atrazina e carbendazim foram quantificados em concentrações de 4-87 ng L ${ }^{-1}$. Machado e colaboradores ${ }^{100}$ avaliaram a presença de contaminantes emergentes, entre os quais, o pesticida atrazina, em amostras de água potável de 22 capitais brasileiras. No método analítico foi utilizado SPE seguida da análise por LC-MS/MS. A recuperação da atrazina foi de $83 \%$ e o LD e LQ foi igual a 1,0 e $2,0 \mathrm{ng} \mathrm{L}^{-1}$, respectivamente. A atrazina foi detectada em $75 \%$ das amostras de água potável em concentrações na faixa de 2,0 a $6,0 \mathrm{ng} \mathrm{L}^{-1}$.

De Souza e colaboradores ${ }^{109}$ avaliaram a presença de 14 pesticidas (ametrina, atrazina, azoxistrobina, carbendazim, carbofurano, clomazona, clorpirifós, diuron, hexazinona, imidacloprido, malation, simazina, tebuconazol e tebutiuron) em água superficial e água de abastecimento público em Londrina, no estado do Paraná, Brasil. Os pesticidas foram extraídos por SPE e avaliados por LC-MS/MS. A recuperação dos analitos foi de $60-115 \%$ e LD e LQ foi de 1-33 e 4-99 $\mathrm{ng} \mathrm{L}^{-1}$, respectivamente. Os pesticidas quantificados nas amostras de água superficial e de abastecimento público foram o carbendazim, imidacloprido, atrazina, diuron e azoxistrobina em concentrações que variaram entre 7,1-265 ng L ${ }^{-1}$. Na água de abastecimento público a frequência de detecção foi de $95 \%$ para o imidacloprido, $58 \%$ para o azoxistrobina, $79 \%$ para o atrazina, $83 \%$ para o diuron e $8 \%$ para o carbendazim. No estado do Rio Grande do Sul, Brasil, foi avaliada a presença de 22 pesticidas e 8 compostos, entre produtos farmacêuticos e de higiene pessoal, em águas superficiais e de abastecimento público. Os pesticidas foram extraídos por SPE e analisados por LC-MS/MS com recuperação entre 49-132\% e LQ entre $0,8-80 \mathrm{ng} \mathrm{L}^{-1}$. A concentração dos pesticidas na água superficial foi de 4-1000 ng L $\mathrm{L}^{-1}$ e na água potável foi de 4-490 ng L-1. A atrazina foi o pesticida mais frequentemente detectado e foi quantificada em concentrações entre 5 e $49 \mathrm{ng} \mathrm{L}{ }^{-1} .^{110}$

\section{ALIMENTOS}

De maneira geral, a determinação de pesticidas em amostras alimentícias envolve as etapas de homogeneização, extração e clean-up, como preparo de amostra, e separação, detecção e análise dos resultados..$^{90}$ As principais técnicas de preparo de amostras empregadas são a LLE, ${ }^{128-131} \mathrm{SPE}^{132-135} \mathrm{e}$ QuEChERS. ${ }^{136-141}$ Além dessas, a SPME, ${ }^{142-144}$ microextração em gota única (SDME, do inglês single drop microextraction), ${ }^{145,146}$ microextração em fluxo contínuo (CFME, do inglês continuous flow microextraction ${ }^{147}$ e extração sólido-líquido (SLE, do inglês solid-liquid extraction). ${ }^{148}$ A complexidade das matrizes adicionam um desafio maior na escolha da melhor técnica de preparo de amostras, no entanto, o analista devem considerar as características das mesmas, bem como as propriedades físico químicas dos pesticidas de interesse e a técnica que será empregada na quantificação, o que impossibilita a elaboração de um protocolo único para todas as amostras e todos os pesticidas. Além disso, para fins de monitoramento e avaliação do risco, é importante que os métodos analíticos apresentem limites de detecção e quantificação inferiores aos LMR ou IDA. Para a separação e detecção dos analitos nas amostras, as técnicas mais empregadas para compostos voláteis são GC, podendo ser acoplada a detectores por captura de elétrons (ECD, do inglês eléctron capture detector), ${ }^{146,149-151}$ detectores de nitrogênio-fósforo (NPD, do inglês nitrogen phosphorus detector), ${ }^{145,152-154}$ detectores MS ${ }^{147,151,155-158}$ e MS/MS. ${ }^{136,159,160}$ Para compostos não voláteis, a principal técnica de separação utilizada é a LC, podendo ser acoplada a detectores de arranjo de diodos (DAD, do inglês diode array detector $)^{161-163}$ e MS/MS..$^{137,157,164-168}$ A Tabela 2 sintetiza algumas técnicas e parâmetros analíticos utilizados para quantificar resíduos de pesticidas em amostras de alimentos.

Silva e Faria, ${ }^{164}$ por exemplo, desenvolveram e validaram um método analítico para quantificar resíduos de 7 pesticidas em amostras de méis produzidos na região do Triângulo Mineiro (Brasil). Utilizaram QuEChERS como preparo de amostra e UHPLC-MS/MS para determinar e quantificar os compostos. O LD do método variou entre 0,6 e $4 \mu \mathrm{g} \mathrm{L}^{-1}$, valores esses inferiores ao LMR estabelecidos para os compostos estudados. A recuperação variou entre 89 e $107 \%$ e precisão reportada com RSD $<14 \%$. O método foi aplicado em 7 amostras de mel, sendo possível quantificar metomil e imidacloprido em todas as amostras analisadas. Segundo os pesquisadores, esses resultados mostram como as colmeias próximas as plantações de grãos e cana-de-açúcar estão sendo afetadas pela aplicação de pesticidas na região de estudo.

\section{MATRIZES BIOLÓGICAS}

Diversos estudos são dedicados à avaliação da exposição externa aos pesticidas, por meio do levantamento da sua ocorrência em diferentes matrizes, como por exemplo, nos alimentos e na água. Porém, além dessa abordagem convencional há um interesse crescente em avaliar a exposição de contaminantes ambientais usando dados de biomonitoramento, ou seja, da dose interna. ${ }^{169} \mathrm{O}$ 
Tabela 2. Técnicas analíticas para determinação de resíduos de pesticidas em amostras de alimentos

\begin{tabular}{|c|c|c|c|c|c|c|}
\hline Matriz & Analitos & $\begin{array}{l}\text { Preparo de } \\
\text { amostra }\end{array}$ & Análise instrumental & $\mathrm{LD}$ & LQ & Referência \\
\hline Frutas e suco de frutas & $\begin{array}{l}\text { Diazinon, malation, imidacloprida, } \\
\text { triadimefon }\end{array}$ & LLE & LC-MS/MS & $0,3-30 \mu \mathrm{g} \mathrm{L}^{-1}$ & & 128 \\
\hline $\begin{array}{l}\text { Maça, uva e suco de } \\
\text { cereja }\end{array}$ & $\begin{array}{c}\text { Diazinon, clorpirifós, fosalona, } \\
\text { ametreina, propazine, simazina, } \\
\text { penconazol, hexazonazol, } \\
\text { diniconazol, difenoconazol, } \\
\text { tebuconazol }\end{array}$ & LLE & $\begin{array}{l}\text { GC-FID } \\
\text { GC-MS }\end{array}$ & $0,34-5 \mu \mathrm{g} \mathrm{L}^{-1}$ & $1-16 \mu g \mathrm{~L}^{-1}$ & 130 \\
\hline Suco de laranja & $\begin{array}{l}\text { Acefato, carbendazim, acetamiprido, } \\
\text { imidacloprida, metrifonato, } \\
\text { ametrina, bromacila, carbofuran, } \\
\text { tiofanato-metílico, diuron, pirimifos- } \\
\text { metila, tebuconazol, diflubenzuron, } \\
\text { malation, diazion, difenoconazole, } \\
\text { clorpirifós, abamectina }\end{array}$ & LLE & LC-ESI-MS/MS & $0,003-0,33 \mathrm{mg} \mathrm{L}^{-1}$ & $0,01-1,11 \mathrm{mg} \mathrm{L}^{-1}$ & 129 \\
\hline Óleo de soja & 95 pesticidas & LLE & GC-MS & & $0,004-0,05 \mathrm{mg} \mathrm{kg}^{-1}$ & 131 \\
\hline $\begin{array}{l}\text { Alface, brócolis, } \\
\text { mostarda, espinafre, } \\
\text { couve-flor, salsão e } \\
\text { repolho }\end{array}$ & $\begin{array}{c}\alpha-\mathrm{HCH}, \beta-\mathrm{HCH}, \gamma-\mathrm{HCH} \text {, clorpirifós, } \\
\text { DDD, DDE, diazinon, dimetoato, } \\
\text { DDT, endosulfan, fenvelerato, } \\
\text { malation, paration metil, permetrina, } \\
\text { paration etil }\end{array}$ & SPE & GC-ECD & $0,02-4,5 \mathrm{ng} \mathrm{g}^{-1}$ & $0,2-45 \mathrm{ng} \mathrm{g}^{-1}$ & 132 \\
\hline Suco de pera & $\begin{array}{c}\alpha-\mathrm{HCH}, \beta-\mathrm{HCH}, \gamma-\mathrm{HCH}, \delta-\mathrm{HCH}, \\
\text { p,p'-DDE, p,p'-DDD, o,p'-DDT, } \\
\text { p,p'-DDT }\end{array}$ & SPE & GC-MS/MS & $0,39-0,7 \mathrm{ng} \mathrm{L}^{-1}$ & $1,45-2 \mathrm{ng} \mathrm{L}^{-1}$ & 133 \\
\hline $\begin{array}{l}\text { Repolho, couve- } \\
\text { flor, goiaba, quiabo, } \\
\text { berinjela, cebola, } \\
\text { maçã, banana, uva, } \\
\text { laranja, romã }\end{array}$ & 60 pesticidas & SPE & UHPLC-TOF/MS & $0,3-3,8 \mu \mathrm{g} \mathrm{kg}^{-1}$ & $0,8-11,8 \mu \mathrm{g} \mathrm{kg}^{-1}$ & 134 \\
\hline Tomate & $\begin{array}{l}\text { Metolcarb, carbofurano, isoprocarbe, } \\
\text { metiocarbe }\end{array}$ & SPE & HPLC-DAD & $0,58-2,06 \mathrm{ng} \mathrm{g}^{-1}$ & & 135 \\
\hline Ervas medicinais & 135 pesticidas & $\begin{array}{c}\text { SPE } \\
\text { QuEChERS }\end{array}$ & LC-MS/MS & & $1-5 \mathrm{ng} \mathrm{g}^{-1}$ & 140 \\
\hline Folhas de alcachofra & 98 pesticidas & QuEChERS & $\begin{array}{c}\text { GC-MS } \\
\text { LC-MS/MS }\end{array}$ & $0,003-0,025 \mathrm{mg} \mathrm{kg}^{-1}$ & & 136 \\
\hline
\end{tabular}

\begin{tabular}{|c|c|c|c|c|c|c|}
\hline Alface & $\begin{array}{l}\text { Acefato, carbaril, carbendazim, } \\
\text { metamidofós, metomil, procloraz, } \\
\text { tebuconazol, azoxistrobina, } \\
\text { clotianidina, difenoconazol, } \\
\text { fenamidone, imidaclopida, } \\
\text { iprodiona, malation, tiaclopride, } \\
\text { tiametoxame }\end{array}$ & QuEChERS & LC-MS/MS & & $5-3200 \mu \mathrm{g} \mathrm{kg}^{-1}$ & 137 \\
\hline Tomate & 57 pesticidas & QuEChERS & LC-ESI-MS/MS & $0,0005-0,02 \mathrm{mg} \mathrm{kg}^{-1}$ & $0,002-0,07 \mathrm{mg} \mathrm{kg}^{-1}$ & 138 \\
\hline Alho-poró, alface & 70 pesticidas & QuEChERS & LC-MS/MS & $0,1-2,4 \mu \mathrm{g} \mathrm{kg}^{-1}$ & $0,3-7,9 \mu \mathrm{g} \mathrm{kg}^{-1}$ & 139 \\
\hline Pepino, pera, repolho & $\begin{array}{l}\text { Bifentrin, fenpropatrina, cialotrina, } \\
\text { permetrina, ciflutrina, flucitrinato, } \\
\text { fenvalerato, deltametrina }\end{array}$ & SPME & GC- $\mu \mathrm{ECD}$ & $0,1-0,5 \mathrm{ng} \mathrm{g}^{-1}$ & $0,3-1,7 \mathrm{ng} \mathrm{g}^{-1}$ & 142 \\
\hline Pera, uva e berinjela & $\begin{array}{c}\text { Diazinon, paration, fentiona, } \\
\text { clorpirifós }\end{array}$ & SPME & GC-IMS & $0,05-0,3 \mu \mathrm{g} \mathrm{Kg}^{-1}$ & & 143 \\
\hline Suco de fruta & $\begin{array}{c}\text { Clorpirifós, penconazol, } \\
\text { procimidona, bromopropilato, } \\
\text { lambda-cialotrina }\end{array}$ & SPME & GC-ECD & $0,002-0,667 \mathrm{ng} \mathrm{mL}^{-1}$ & & 144 \\
\hline Chá & $\begin{array}{l}\text { Forato, diazinon, tolclofos-metil, } \\
\text { malation, fentiona, quinalfós, } \\
\text { fenamifós }\end{array}$ & SDME & GC-MS & $0,4-1,7 \mu \mathrm{g} \mathrm{Kg}^{-1}$ & $1,1-5,6 \mu \mathrm{g} \mathrm{Kg}^{-1}$ & 145 \\
\hline Mel & $\begin{array}{l}\text { HCB, diazinon, lindane, clorpirifós- } \\
\text { metil, clorpirifós-etil, procimidona, } \\
\text { captan, } \alpha \text {-endosulfan, } \beta \text {-endosunfan, } \\
\text { endosunfan sulfato, bromopropilato, } \\
\text { fosalona, fenarimol, coumafós }\end{array}$ & SDME & GC-MS & $0,01-3,75 \mu \mathrm{g} \mathrm{Kg}^{-1}$ & & 146 \\
\hline
\end{tabular}


Tabela 2. Técnicas analíticas para determinação de resíduos pesticidas em amostras de alimentos (cont.)

\begin{tabular}{|c|c|c|c|c|c|c|}
\hline Matriz & Analitos & $\begin{array}{c}\text { Preparo de } \\
\text { amostra }\end{array}$ & Análise instrumental & LD & LQ & Referência \\
\hline Vegetais & $\begin{array}{c}\text { Demeton-s-metil, forato, diazinon, } \\
\text { tolclofos-metil, malation, fentiona, } \\
\text { quinalfós, fenamifós }\end{array}$ & CFME & GC-MS & $0,59-1,57 \mu \mathrm{g} \mathrm{Kg}^{-1}$ & $1,96-5,23 \mu \mathrm{g} \mathrm{Kg}^{-1}$ & 147 \\
\hline Alface & Procimidona, iprodiona & SLE & GC-ECD & $0,4-37 \mu \mathrm{g} \mathrm{Kg}^{-1}$ & & 148 \\
\hline
\end{tabular}

LD: limite de detecção; LQ: limite de quantificação; SPE: extração em fase sólida (do inglês, solid-phase extraction); LLE: líquido-líquido (do inglês, liquid-liquid extraction); SLE: extração sólido-líquido (do inglês, solid-liquid extraction); SPME: microextração em fase sólida (do inglês, solid-phase microextraction); SDME: microextração em gota única (do inglês, single drop microextraction); CFME: microextração em fluxo contínuo (do inglês, continuous flow microextraction); GC: cromatografia gasosa (do inglês, gas chromatography); LC: cromatografia líquida (do inglês, liquid chromatography); UHPLC: cromatografia líquida de ultra eficiência (do inglês, ultra high performance liquid chromatography); HPLC: cromatografia líquida de alta eficiência (do inglês, ultra high performance liquid chromatography); MS: espectrometria de massas (do inglês, mass spectrometry); MS/MS: espectrometria de massas sequencial (do inglês, tandem mass spectrometry); TOF: tempo de voo (do inglês, time of flight); DAD: detector de arranjo de diodos (do inglês; diode array detector); ECD: detector de captura de elétrons (do inglês, electron capture detector); FID: detector por ionização de chama (do inglês, flame ionization detector); IMS: espectrometria de mobilidade iônica (do inglês, ion mobility spectrometry)

biomonitoramento é definido como uma medição direta da exposição humana aos contaminantes por meio da substância química e/ou dos seus metabólitos presentes em amostras biológicas. ${ }^{170,171}$

A seleção da amostra biológica para o biomonitoramento humano é determinada pelas propriedades químicas e da farmacocinética da substância que está sendo monitorada. $\mathrm{O}$ sangue e a urina são considerados matrizes convencionais no biomonitoramento, porém, nos últimos anos tem aumentado o interesse por matrizes alternativas, tais como cabelo, mecônio, leite materno, entre outras..$^{91,172,173}$ O sangue é a matriz ideal para a determinação da maioria dos produtos químicos devido ao contato do plasma sanguíneo com todo o organismo e ao seu equilíbrio com órgãos e tecidos onde os compostos são armazenados. A principal desvantagem do uso do sangue no biomonitoramento humano é por ele ser uma matriz invasiva e, portanto, poder haver uma resistência maior na resposta dos participantes em estudos epidemiológicos voluntários, além disso, sua disponibilidade geralmente é limitada e a coleta necessita de pessoal qualificado. Em geral, o sangue e seus componentes (soro e plasma) são preferidos na determinação de pesticidas persistentes, como os organoclorados, que têm um tempo de meia-vida mais longo nessas matrizes. ${ }^{9,91,174}$

A urina é uma matriz representativa da exposição recente, pois pesticidas que não são persistentes, como piretróides e organofosforados, são rapidamente metabolizados e eliminados em poucos dias. Além disso, a urina é uma matriz não invasiva, abundante e fácil de coletar. As amostras de urina podem ser pontuais ou de 24 horas. A coleta de amostras pontuais é mais fácil e por isso é realizada com mais frequência, porém, para a compensação de variações no volume e na concentração dos analitos, que ocorrem nesse tipo de amostra, é necessário realizar um ajuste, sendo o ajuste da concentração da creatinina o mais empregado. ${ }^{91,172,173,175,176}$ Como vários metabólitos dos organofosforados, piretróides ou neonicotinóides são, em parte, excretados na urina como conjugados de glicuronídeo e sulfato, geralmente é realizado um pré-tratamento nas amostras por meio da desconjugação por hidrólise enzimática ou ácida com $\beta$-glucoronidase/sulfatase. ${ }^{91,177,178}$

O cabelo humano é uma matriz estável que apresenta diversas vantagens, tais como, fácil coleta, transporte e armazenamento, além de fornecer informações sobre as exposições a curto e longo prazo. No entanto, existem algumas dificuldades na diferenciação da deposição endógena (dose interna por difusão de capilares sanguíneos) e exógena (ambiente externo) dos contaminantes. Para a remoção da gordura, suor e das substâncias exógenas presentes na superfície do cabelo é realizada uma etapa de descontaminação com água e solventes orgânicos, como a acetonitrila, ou soluções de surfactantes, como o dodecil sulfato de sódio. Para aumentar a extração dos analitos, de modo geral, é realizado um pré-tratamento por meio da incubação ou decomposição enzimática, alcalina ou ácida do cabelo. Os inseticidas organoclorados, piretróides e organofosforados foram investigados no cabelo, principalmente por meio da análise dos seus compostos originais..$^{91,173,179,180}$

O leite materno é comumente empregado no monitoramento dos níveis de exposição da mãe e do bebê por meio da amamentação, sendo mais utilizado no monitoramento de pesticidas lipofílicos devido aos seus elevados teores de gordura. Para comparar os níveis de pesticidas, um ajuste de lipídios é necessário, uma vez que a concentração de lipídios no leite humano não é constante. ${ }^{91,181}$ Pesticidas organoclorados, como DDT e $\mathrm{HCH}$, foram detectados em amostras de leite materno. ${ }^{182} \mathrm{O}$ mecônio é apropriado para medir a exposição pré-natal aos pesticidas, pois eles se acumulam a partir do terceiro mês de gestação até o nascimento. As principais vantagens dessa matriz são a sua fácil coleta, a quantidade de amostra que pode ser coletada e as informações sobre a exposição de longo prazo do feto. ${ }^{173,183}$ Ostrea e colaboradores ${ }^{184}$ analisaram a presença de diversos pesticidas organoclorados no mecônio e pela comparação desta matriz com o cabelo infantil e cordão umbilical, eles concluíram que o mecônio era a melhor matriz para avaliação da exposição fetal a pesticidas. A Figura 5 mostra os tempos de vida dos pesticidas persistentes e não persistentes no sangue humano, urina e mecônio fetal.

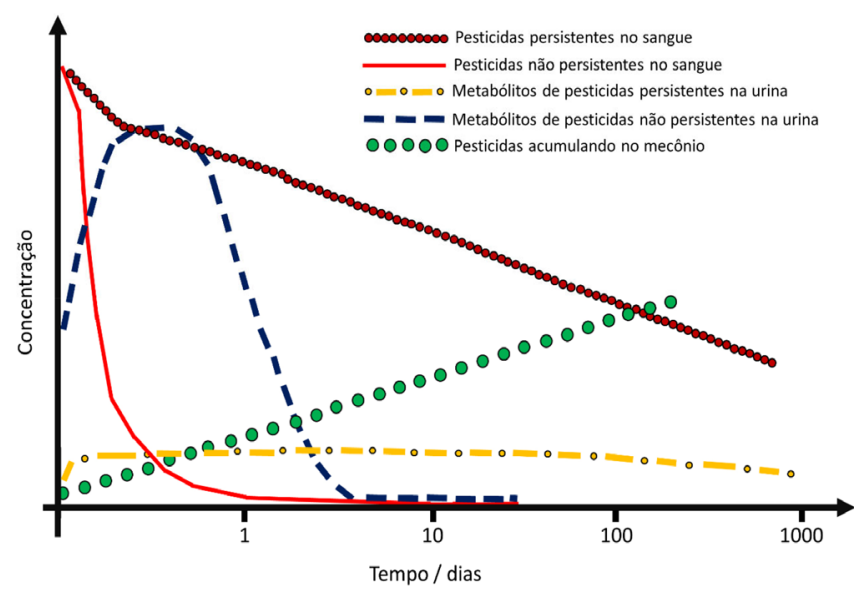

Figura 5. Esquema do tempo de vida e do destino característico dos pesticidas persistentes e não persistentes no sangue humano, urina e mecônio fetal (Adaptado de Barr e Needham ${ }^{9}$ 
As técnicas mais empregadas na extração de pesticidas de amostras biológicas são a SLE, ${ }^{45,179,183,185-187}$ LLE, ${ }^{188-192}$ SPE nas configurações off-line ${ }^{169,177,187,193-196}$ e on-line, ${ }^{197-201}$ SPME $^{188,202-206}$ e QuEChERS. ${ }^{195,207-211}$ Para a avaliação dos biomarcadores de pesticidas as técnicas mais empregadas são a cromatografia gasosa ou líquida acopladas à espectrometria de massas. Nas análises realizadas por LC, o sistema mais utilizado é o LC acoplado à espectrometria de massas em tandem (MS/MS). ${ }^{91}$ A Tabela 3 apresenta algumas técnicas e os principais parâmetros analíticos empregados na determinação de pesticidas em amostras biológicas humanas.

Tao e colaboradore ${ }^{177}$ otimizaram um método para o monitoramento biológico do imidacloprido e seu principal metabólito, o ácido 6-cloronicotínico (6-CNA), em amostras de urina de aplicadores de pesticidas. A extração dos analitos foi avaliada por LLE, QuEChERS e SPE com os cartuchos Polar Enhanced Polymer (PEP), Polymer
Cation eXchange (PCX) e PolymerWeak Cation eXchange (PWCX) e a análise foi realizada por LC-MS/MS. A SPE com o cartucho PEP obteve a melhor extração dos analitos, com recuperações médias entre 78,3-109,8\% e os limites de detecção e quantificação foram de $0,009-0,011 \mu \mathrm{g} \mathrm{L}-1$ e $0,029-0,038 \mu \mathrm{g} \mathrm{L}^{-1}$, respectivamente. Nas amostras de urina, a concentração de imidacloprido e 6-CNA antes da aplicação do pesticida foi de 0,57-8,91 $\mu \mathrm{g} \mathrm{L}^{-1} \mathrm{e} 0,21-2,92 \mu \mathrm{g} \mathrm{L} \mathrm{L}^{-1}$, respectivamente, e após a aplicação foi de 0,38-24,58 $\mu \mathrm{g} \mathrm{L}^{-1}$ e $0,11-6,88 \mu \mathrm{g} \mathrm{L}^{-1}$, respectivamente. Lehman e colaboradores ${ }^{207}$ desenvolveram um método QuEChERS modificado para análise multirresíduo de 37 pesticidas em cabelo humano por GC-MS e UPLC-MS/MS. O sorvente Supel ${ }^{\mathrm{TM}} \mathrm{QuE}$ Z-Sep+ obteve uma recuperação entre $40-150 \%$ para 28 pesticidas. Os limites de detecção variaram de 0,2 a $86,6 \mathrm{pg} \mathrm{mg}^{-1}$ e de 0,5 a 6,3 pg $\mathrm{mg}^{-1}$ para substâncias sensíveis a GC e UPLC, respectivamente. O método foi avaliado em amostras de cabelo de voluntários $(\sim 300 \mathrm{mg})$

Tabela 3. Metodologias para determinação de pesticidas em amostras biológicas humanas

\begin{tabular}{|c|c|c|c|c|c|c|}
\hline Matriz & Pesticidas & $\begin{array}{l}\text { Preparo de } \\
\text { amostra }\end{array}$ & Análise instrumental & LD & LQ & Referência \\
\hline Urina & Glifosato e AMPA & $\begin{array}{l}\text { SPE com ponta } \\
\text { de pipeta }\end{array}$ & LC-HRMS & $1-3 \mu \mathrm{g} \mathrm{L} \mathrm{L}^{-1}$ & $2,5-6 \mu \mathrm{g} \mathrm{L} \mathrm{L}^{-1}$ & 193 \\
\hline Urina & Imidacloprida e ácido 6-cloronicotínico & SPE & LC-MS/MS & $0,009-0,011 \mu \mathrm{g} \mathrm{L}^{-1}$ & $0,029-0,038 \mu \mathrm{g} \mathrm{L}^{-1}$ & 177 \\
\hline Urina & $\begin{array}{c}\text { Metabólitos de organofosforados (TCPY, } \\
\text { IMPY, PNP, CMHC, DEAMPY) e de } \\
\text { piretróides (3-PBA, 4-F-3-PBA, } \\
\text { cis/trans-DCCA) }\end{array}$ & $\begin{array}{l}\text { Cromatografia de } \\
\text { fluxo turbulento }\end{array}$ & LC- HRMS & & $1-10 \mu g \mathrm{~L}^{-1}$ & 198 \\
\hline Urina & $\begin{array}{c}\text { Metabólitos de organofosforados (DAPs, } \\
\text { TCPY, PNP, MDA, CMHC, IMPY, } \\
\text { DEAMPY) e de piretróides } \\
\text { (3-PBA, 4-F-3-PBA) }\end{array}$ & SPE & UHPLC-MS/MS & $14-69 \mu g \mathrm{~L}^{-1}$ & & 196 \\
\hline Urina & Glifosato & SPE & LC-MS/MS & & $0,5 \mu \mathrm{g} \mathrm{L}^{-1}$ & 175 \\
\hline Sangue & $\begin{array}{c}\text { Carbofurano, terbufos, carbaril, } \\
\text { metiocarbe, pirimicarb }\end{array}$ & SPE dispersiva & PTV-LVI-GC/MS & & $0,02-0,03 \mathrm{mg} \mathrm{L}^{-1}$ & 214 \\
\hline Sangue & $\alpha$-HCH, $\beta$-HCH, $\gamma$-HCH, DDE & SPE online & LVI-GC-HRMS & & $0,007-0,017 \mu \mathrm{g} \mathrm{L}^{-1}$ & 200 \\
\hline Sangue & $\begin{array}{c}\text { Amitraz e seus metabolitos (DMPF, DMF, } \\
\text { DMA) }\end{array}$ & SPE & LC-MS/MS & $0,5 \mu \mathrm{g} \mathrm{L}^{-1}$ & $2 \mu g \mathrm{~L}^{-1}$ & 215 \\
\hline $\begin{array}{l}\text { Sangue do } \\
\text { cordão umbilical }\end{array}$ & Cipermetrina e clorpirifós & SPE online & LC-MS/MS & $0,01-0,05 \mu \mathrm{g} \mathrm{L}^{-1}$ & & 197 \\
\hline Sérum & $\begin{array}{c}\alpha-\mathrm{HCH}, \beta-\mathrm{HCH}, \gamma-\mathrm{HCH}, \delta \text {-HCH, } \\
\text { 4,4'-DDD, DDE, DDT, aldrin, heptacloro, } \\
\text { heptacloro epóxido, } \alpha \text {-endosulfan, } \\
\beta \text {-endosulfan, sulfato endosulfan, HCB, } \\
\text { atrazina }\end{array}$ & SPME & GC-MS & $0,22-5,41 \mu \mathrm{g} \mathrm{L}^{-1}$ & $1,57-10,09 \mu \mathrm{g} \mathrm{L}^{-1}$ & 204 \\
\hline Plasma & $\begin{array}{l}\mathrm{HCB}, \text { trans-clordano, cis-clordano, } \\
\text { trans- nonacloro, } \mathrm{p}, \mathrm{p}^{\prime} \text {-DDE }\end{array}$ & SPE & GC-HRMS & $5,9-89,1 \mu \mathrm{g} \mathrm{L}^{-1}$ & & 216 \\
\hline Cabelo & $\begin{array}{c}\text { Acetoclor, } \alpha \text {-clordano, } \gamma \text {-clordano, } \\
\text { nonacloro, oxiclordano, clorpirifós- } \\
\text { etil, clorpirifós-metil, } \lambda \text {-cialotrina, } \\
\alpha \text {-cipermetrina, } \beta \text {-cipermetrina, } \\
\text { deltametrina, diazinon, dieldrin, } \\
\alpha \text {-endosulfan, heptacloro epóxido A, } \\
\text { heptacloro epóxido B, metoxicloro, } \\
\text { p,p'-DDE, p,p'-DDT, o,p'-DDT, } \\
\text { p,p'-DDD, acetamiprido, atrazina, DIA, } \\
\text { DEA, carbofurano, imidacloprida, linuron }\end{array}$ & QuEChERS & $\begin{array}{c}\text { GC-MS } \\
\text { UHPLC-MS/MS }\end{array}$ & $0,18-86,6 \mu \mathrm{g} \mathrm{g}^{-1}$ & $0,6-288,5 \mu \mathrm{g} \mathrm{g}^{-1}$ & 207 \\
\hline Cabelo & $\begin{array}{l}\text { Dimetomorfo, penconazol, ciazofamida, } \\
\text { fenamidona, quinoxifena, clorpirifós, } \\
\text { ciprodinil, metrafenona, boscalid, } \\
\text { pirimetanil, tebuconazol, azoxistrobina, } \\
\text { fenhexamida, imidacloprida, carbendazim, } \\
\text { imazalil, tiabendazol, piraclostrobina, } \\
\text { difenoconazol, metolaclor, tiaclopride, } \\
\text { dimetenamida, fluopirame, procloraz, } \\
\text { cialotrina (lambda), propamocarb, } \\
\text { cloridazon }\end{array}$ & SLE & LC-MS/MS & $0,5-15 \mu \mathrm{g} \mathrm{g}^{-1}$ & & 45 \\
\hline
\end{tabular}


Tabela 3. Metodologias para determinação de pesticidas em amostras biológicas humanas (cont.)

\begin{tabular}{|c|c|c|c|c|c|c|}
\hline Matriz & Pesticidas & $\begin{array}{c}\text { Preparo de } \\
\text { amostra }\end{array}$ & Análise instrumental & LD & LQ & Referência \\
\hline Cabelo & $\begin{array}{c}\text { Aldrin, cis-clordano, trans-clordano, } \\
\text { p,p'- DDD, o,p'-DDD, p,p'- DDE, } \\
\text { o,p'-DDE, p,p'- DDT, o,p'-DDT, dieldrin, } \\
\text { endosulfan I, endosulfan II, endrin, } \\
\text { heptacloro, heptacloro epóxido A, } \\
\text { heptacloro epóxido B, hexaclorobenzeno, } \\
\alpha \text {-HCH, } \beta \text {-HCH, } \gamma \text {-HCH, } \delta \text {-HCH, } \\
\text { metoxicloro, isodrin, oxiclordano, mirex }\end{array}$ & SLE & GC-MS & & $0,7-33 \mathrm{pg} \mathrm{g}^{-1}$ & 217 \\
\hline Cabelo & Penconazol e tebuconazol & SLE & LC-MS/MS & & $1 \mu \mathrm{g} \mathrm{g}^{-1}$ & 218 \\
\hline Urina e cabelo & $\begin{array}{c}\text { Metabólitos de organofosforados (DMP, } \\
\text { DEP, DETP, DEDTP) }\end{array}$ & $\begin{array}{l}\text { SPE } \\
\text { SLE }\end{array}$ & $\begin{array}{l}\text { UHPLC-MS/MS } \\
\text { GC-MS }\end{array}$ & $\begin{array}{l}0,5-1 \mu \mathrm{g} \mathrm{L}^{-1} \\
3-6 \mathrm{pg} \mathrm{g}^{-1} \\
\end{array}$ & & 187 \\
\hline Mecônio & $\begin{array}{c}\text { Malation, diazinon, clorpirifós, } \\
\text { cipermetrina, ciflutrina, deltametrina, } \\
\text { propoxur, mancozeb, isoproturon, MCPA } \\
\text { e os metabólitos DMP, DMTP, DMDTP, } \\
\text { DMDTP, DEP, DETP, DCCA, EU, ETU, } \\
\text { desmetil isoproturon }\end{array}$ & SLE & LC-MS/MS & $0,07-66,7 \mathrm{ng} \mathrm{g}^{-1}$ & $0,2-200 \mathrm{ng} \mathrm{g}^{-1}$ & 186 \\
\hline Mecônio & $\begin{array}{c}\text { Clordano, clorpirifós, DDT, } \\
\text { diazinon, lindane, malation, paration, } \\
\text { pentaclorofenol }\end{array}$ & SLE & GC-MS & $0,186-6,616 \mathrm{mg} \mathrm{L}^{-1}$ & & 219 \\
\hline Mecônio & $\begin{array}{c}\text { Clorpirifós, DETP, diazinon, DEP, } \\
\text { malation, DMP, propoxur, ETU, } \\
\text { isoproturon, desmetil isoproturon, MCPA }\end{array}$ & SLE & LC-MS/MS & $0,1-1 \mathrm{ng} \mathrm{g}^{-1}$ & & 220 \\
\hline Leite materno & $\begin{array}{c}\text { o, } \mathrm{p}^{\prime}-\mathrm{DDE}, \mathrm{p}, \mathrm{p}^{\prime}-\mathrm{DDE}, \mathrm{o}, \mathrm{p}^{\prime}-\mathrm{DDD}, \\
\mathrm{p}, \mathrm{p}^{\prime}-\mathrm{DDD}, \mathrm{o}, \mathrm{p}^{\prime}-\mathrm{DDT}, \mathrm{p}, \mathrm{p}^{\prime}-\mathrm{DDT}, \alpha-\mathrm{HCH}, \\
\beta \text {-HCH, } \gamma \text {-HCH, } \delta \text {-HCH, oxiclordano, } \\
\text { trans-clordano, trans-nonaclordano, } \\
\text { cis-nonaclordano, HCB, heptacloro, } \\
\text { heptacloro epóxido, mirex }\end{array}$ & SPE & GC-HRMS & $0,0004-0,0010 \mathrm{ng} \mathrm{g}^{-1}$ & $0,10-0.25 \mathrm{ng} \mathrm{g}^{-1}$ & 221 \\
\hline Leite materno & $\begin{array}{c}\alpha \text {-HCH, } \beta \text {-HCH, } \gamma \text {-HCH, } \delta \text {-HCH, } \\
\varepsilon \text {-HCH, 4,4'-DDT, 2,4'-DDT, 4,4'-DDD, } \\
\text { 2,4'-DDD, 4,4'-DDE, 2,4'-DDE, trans- } \\
\text { clordano, cis-clordano, oxy-clordano, } \\
\text { heptacloro, cis- heptaclorepóxido, } \\
\text { trans- heptaclorepóxido, aldrin, dieldrin, } \\
\text { endrin, endosulfan-I, endosulfan-II, } \\
\text { metoxicloro, mirex, pentaclorobenzeno, } \\
\text { hexaclorobenzeno, pentacloroanisol, } \\
\text { octacloroestireno }\end{array}$ & LLE & GC-HRMS & $1-31 \mathrm{pg} \mathrm{g}^{-1}$ & & 190 \\
\hline Leite materno & $\begin{array}{c}\alpha \text {-HCH, } \beta \text {-HCH, } \gamma \text {-HCH, } \delta \text {-HCH, } \\
\text { heptacloro, } \alpha \text {-clordano, } \gamma \text {-clordano, } \\
\text { 4,4'-DDT, 4,4'-DDE, 4,4'-DDD }\end{array}$ & LLE & GC-ECD & $8 \mathrm{ng} \mathrm{g}^{-1}$ & $17 \mathrm{ng} \mathrm{g}^{-1}$ & 222 \\
\hline
\end{tabular}

LD: limite de detecção; LQ: limite de quantificação; SPE: extração em fase sólida (do inglês, solid-phase extraction); LLE: líquido-líquido (do inglês, liquidliquid extraction); SLE: extração sólido-líquido (do inglês, solid-liquid extraction); SPME: microextração em fase sólida (do inglês, solid-phase microextraction); PTV: temperatura programável de vaporização (do inglês, programmed temperature vaporization); LVI: injeção de grande volume (do inglês, large volume injection); GC: cromatografia gasosa (do inglês, gas chromatography); LC: cromatografia líquida (do inglês, liquid chromatography); UHPLC: cromatografia líquida de ultra eficiência (do inglês, ultra high performance liquid chromatography); MS: espectrometria de massas (do inglês, mass spectrometry); MS/MS: espectrometria de massas sequencial (do inglês, tandem mass spectrometry); HRMS: espectrometria de massas de alta resolução (do inglês, high resolution mass spectrometry); ECD: detector de captura de elétrons (do inglês, electron capture detector).

que viviam em uma área de produção de vegetais em Burkina Faso. A presença de 8 pesticidas foram detectadas nas amostras, sendo estes, acetamiprido, gamma-trans-chlordane, clorpirifós, $\lambda$-cyhalothrin, deltametrina, dieldrin, imidacloprido e p,p'-DDE, em concentrações entre 2,2 e $369,4 \mathrm{pg} \mathrm{mg}^{-1}$.

Du e colaboradores ${ }^{182}$ utilizaram QuEChERS e GC-MS/MS para avaliar a presença de 88 pesticidas no leite materno. O método obteve uma excelente recuperação (70-120\%) e limites de detecção entre $0,2-2,0 \mu \mathrm{g} \mathrm{L}^{-1}$. Nas amostras de leite foram detectados os pesticidas organoclorados p,p'-DDE, p,p'-DDT e $\beta$-HCH em concentrações

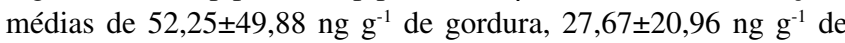
gordura e 48,00 $\pm 22,46 \mathrm{ng} \mathrm{g}^{-1}$ de gordura, respectivamente. FloresRamírez e colaboradores ${ }^{204}$ desenvolveram um método utilizando
SPME para a determinação de Poluentes Orgânicos Persistentes (POPs) em soro humano por GC-MS. Os POPs avaliados foram 14 bifenila policloradas (PCBs), 14 pesticidas organoclorados e a atrazina. A extração dos analitos foi realizada com HeadspaceSPME e a fibra de PDMS apresentou a melhor recuperação para os analitos (70-120\%). Os LD e LQ variaram de 0,24-5,41 $\mu \mathrm{g} \mathrm{L}^{-1}$ e 2,38-10,09 $\mu \mathrm{g} \mathrm{L}^{-1}$, respectivamente. O método foi aplicado em amostras de soro humano de crianças e os pesticidas detectados foram $\mathrm{HCB}\left(2,61-3,4 \mu \mathrm{g} \mathrm{L} \mathrm{L}^{-1}\right), \gamma-\mathrm{HCH}\left(1,53-2,18 \mu \mathrm{g} \mathrm{L}^{-1}\right), \delta-\mathrm{HCH}$ $\left(19,9-77,5 \mu \mathrm{g} \mathrm{L}^{-1}\right)$, DDD $\left(0,2 \mu \mathrm{g} \mathrm{L}^{-1}\right)$, DDE $\left(2,0-52,2 \mu \mathrm{g} \mathrm{L}^{-1}\right) \mathrm{e}$ $\beta$-endosulfan $\left(2,8-7,5 \mu \mathrm{g} \mathrm{L}^{-1}\right)$, endosulfan sulfate $\left(4,4-9,42 \mu \mathrm{g} \mathrm{L}^{-1}\right)$.

Estudos vem sendo realizados para a determinação de pesticidas em amostras biológicas de pessoas intoxicadas por pesticidas. Luzardo 
e colaboradores ${ }^{212}$ desenvolveram um método utilizando LLE para a avaliação simultânea de 109 pesticidas no sangue humano por GCMS/MS e LC-MS/MS. A recuperação dos analitos foi entre $68-105 \%$ e os LDs foram de 0,003-0,050 $\mathrm{mg} \mathrm{L}^{-1}$. O método foi avaliado em amostras de sangue de 2 casos, um envolvendo uma fatalidade de autoenvenenamento e outro na tentativa de homicídio não fatal. No caso do auto-envenenamento, o inseticida organofosforado diazinon foi encontrado na concentração de $6,48 \mathrm{mg} \mathrm{L}^{-1}$ e na tentativa de homicídio o inseticida carbamato aldicarbe foi detectado em uma concentração de $2,32 \mathrm{mg} \mathrm{L}^{-1}$. Mee-Jung e colaboradores ${ }^{213}$ avaliaram as concentrações no sangue pós-morte dos pesticidas organofosforados clorpirifós, diazinon, malation e paration com SPE offline e GC-MS. A recuperação foi acima de $70 \%$ para todos os compostos e os LQ foram entre $0,01-0,05 \mathrm{mg} \mathrm{L}^{-1}$ e $0,003-0,050 \mathrm{mg} \mathrm{L}^{-1}$ por LC e GC, respectivamente. As concentrações médias do clorpirifós, diazinon, malation e paration nas amostras de sangue foram 0,$72 ; 1,03 ; 0,82 \mathrm{e}$ $2,90 \mathrm{mg} \mathrm{L}^{-1}$, respectivamente.

\section{CONCLUSÃO}

A avaliação do impacto dos pesticidas na saúde humana ainda é um desafio, pois a correlação entre seu uso e os efeitos na saúde é muito complexa. A maioria dos estudos que relacionam os efeitos dos pesticidas na saúde humana são realizados no ambiente ocupacional, uma vez que as pessoas que manipulam e/ou aplicam pesticidas são expostas a uma quantidade maior destes contaminantes e, portanto, a avaliação do impacto nesses ambientes é mais facilmente evidenciada.

A química trouxe importantes avanços nas técnicas analíticas para a identificação e quantificação dos pesticidas nas matrizes ambientais, biológicas e alimentícias.

No entanto, estudos que abordam a exposição ambiental aos pesticidas, a qual a maioria da população está submetida, para inferir os riscos à saúde humana empregando análises de amostras como água, alimentos, etc. enfrentam a complexidade da associação direta entre dose e efeito.

Diversos órgãos governamentais discordam sobre a toxicidade dos pesticidas em seres humanos, todavia não se pode ignorar seus possíveis efeitos e, por isso, os estudos observacionais, in vivo e in vitro, são muito importantes para fazer essas associações.

A determinação da exposição interna dos seres humanos (biomonitoramento) e externa (exposição ambiental e alimentícia) são importantes ferramentas, já que os dados obtidos nesses estudos podem ser usados, em conjunto com os estudos observacionais, in vivo e in vitro, na avaliação e gestão de risco para subsidiar políticas públicas voltadas para ele, que é um nicho importante do ponto de vista socioeconômico em todo o mundo.

\section{AGRADECIMENTOS}

Os autores agradecem ao Instituto Nacional de Ciências e Tecnologias Analíticas Avançadas - INCTAA (FAPESP Proc. 2014/50951-4 e CNPq Proc. 465768/2014-8) pelo auxílio financeiro, ao Conselho Nacional de Desenvolvimento Científico e Tecnológico pela bolsa de doutorado (Proc. 142467/2018-9) e à Coordenação de Aperfeiçoamento de Pessoal de Nível Superior pela bolsa de doutorado (Proc. 88887.169503/2018-00).

\section{REFERÊNCIAS}

1. https://www.who.int/topics/pesticides/en/, acessada em dezembro 2020.

2. Kim, K.-H.; Kabir, E.; Jahan, S. A.; Sci. Total Environ. 2017, 575, 525.

3. http://www.planalto.gov.br/ccivil_03/LEIS/L7802.htm, acessada em dezembro 2020 .
4. http://bi.agricultura.gov.br/reports/rwservlet?agrofit_cons\&ingredientes_ consolidado.rdf\&p_id_ingrediente_ativo $=\& p \_n m \_c o m u m \_p o r t=\& p \_i d \_$ grupo_quimico $=\& p \_i d \_c l a s s e=\& p \_i d \_c u l t u r a=\& p \_t i p o \_r e l a t o r i o=C O N$ SOLIDADO\&paramform=no, acessada em dezembro 2020.

5. http://bi.agricultura.gov.br/reports/rwservlet?agrofit_cons\&produtos_ consolidado.rdf\&p_id_produto_formulado_tecnico $=\& p \_n m \_m a r c a \_$ comercial $=\& p \_i d \_r e g i s t r a n t e \_e m p r e s a=\& p \_n r \_r e g i s t r o=\& p \_i d$ ingrediente_ativo $=\&$ p_id_tecnica_aplicacao $=\& p \_i d \_c l a s s e=\& p$ id_classificacao_tox $=\&$ p_id_classificacao_amb $=\&$ p_tipo_ aplicacao $=C \& p \_i d \_c u l t u r a=\& p \_i d \_c u l t u r a \_i n s e t o=\& p \_i d \_c u l t u r a \_$ planta $=\&$ p_id_planta_daninha $=\&$ p_id_cultura_praga $=\&$ p_tipo_ relatorio=CONSOLIDADO\&p_origem_report $=\mathrm{WEB} \&$ paramform $=$ no, acessada em dezembro 2020

6. Teerlink, J.; Hernandez, J.; Budd, R.; Sci. Total Environ. 2017, 599-600, 960.

7. Raeppel, C.; Salquèbre, G.; Millet, M.; Appenzeller, B. M. R.; Sci. Total Environ. 2016, 544, 845.

8. Machado, S. C.; Martins, I.; Regul. Toxicol. Pharmacol. 2018, 98, 276.

9. Barr, D. B.; Needham, L. L.; J. Chromatogr. B 2002, 778, 5.

10. Carson, R.; Silent Spring, Houghton Mifflin: Boston, 1962.

11. Taiwo, A. M.; Chemosphere 2019, 220, 1126.

12. Colosio, C.; Rubino, F. M.; Moretto, A. In International Encyclopedia of Public Health, 2nd ed, Elsevier, 2017.

13. Alcântara, D. B.; Fernandes, T. S. M.; Nascimento, H. O.; Lopes, A. F.; Menezes, M. G. G.; Lima, A. C. A.; Carvalho, T. V.; Grinberg, P.; Milhome, M. A. L.; Oliveira, A. H. B.; Becker, H.; Zocolo, G. J.; Nascimento, R. F.; Food Chem. 2019, 298, 124958.

14. Soler, C.; Hamilton, B.; Furey, A.; James, K. J.; Mañes, J.; Picó, Y.; Anal. Chim. Acta 2006, 571, 1.

15. Parween, T.; Jan, S.; Ecophysiology of Pesticides, $1^{\text {st }}$ ed., Elsevier: Amsterdam, 2019.

16. Perry, E. D.; Moschini, G. C.; J. Environ. Econ. Manage. 2020, 102, 102320.

17. https://sinitox.icict.fiocruz.br/, acessada em dezembro 2020.

18. de Araújo Nascimento, F.; Alves, A. A.; Nunes, H. F.; Miziara, F.; Parise, M. R.; de Melo e Silva, D.; Environ. Sci. Pollut. Res. 2020.

19. Gunnell, D. J.; Eddleston, M.; Int. J. Epidemiol. 2003, 32, 902.

20. Elahi, E.; Weijun, C.; Zhang, H.; Nazeer, M.; Land Use Policy 2019, 83, 461.

21. Caldas, E. D. Pesticide Poisoning in Brazil; Elsevier Inc.: Amsterdam, 2016.

22. Sabarwal, A.; Kumar, K.; Singh, R. P.; Environ. Toxicol. Pharmacol. 2018, 63, 103.

23. Corcino, C. O.; Teles, R. B. de A.; Almeida, J. R. G. da S.; Lirani, L. da S.; Araújo, C. R. M.; Gonsalves, A. de A.; Maia, G. L. de A.; Ciência \& Saúde Coletiva 2019, 24, 3117.

24. García-García, C. R.; Parrón, T.; Requena, M.; Alarcón, R.; Tsatsakis, A. M.; Hernández, A. F.; Life Sci. 2016, 145, 274.

25. Van Bruggen, A. H. C.; He, M. M.; Shin, K.; Mai, V.; Jeong, K. C.; Finckh, M. R.; Morris, J. G.; Sci. Total Environ. 2018, 616-617, 255.

26. Bilal, M.; Iqbal, H. M. N.; Barceló, D.; Sci. Total Environ. 2019, 695, 133896.

27. Sauret, N.; Wortham, H.; Strekowski, R.; Herckès, P.; Nieto, L. I.; Environ. Pollut. 2009, 157, 303.

28. Climent, M. J.; Coscollà, C.; López, A.; Barra, R.; Urrutia, R.; Sci. Total Environ. 2019, 662, 646.

29. dos Santos Neto, A. J.; Siqueira, M. E. P. B.; Quim. Nova 2005, $28,747$.

30. Montagner, C.; Sodré, F.; Acayaba, R.; Vidal, C.; Campestrini, I.; Locatelli, M.; Pescara, I.; Albuquerque, A.; Umbuzeiro, G.; Jardim, W.; J. Braz. Chem. Soc. 2018, 30, 614.

31. Westlund, P.; Yargeau, V.; Sci. Total Environ. 2017, 607-608, 744.

32. Münze, R.; Hannemann, C.; Orlinskiy, P.; Gunold, R.; Paschke, A.; 
Foit, K.; Becker, J.; Kaske, O.; Paulsson, E.; Peterson, M.; Jernstedt, H.; Kreuger, J.; Schüürmann, G.; Liess, M.; Sci. Total Environ. 2017, 599-600, 387.

33. https://portalarquivos2.saude.gov.br/images/pdf/2018/marco/29/PRC5-Portaria-de-Consolida----o-n---5--de-28-de-setembro-de-2017.pdf, acessada em dezembro 2020.

34. http://www2.mma.gov.br/port/conama/legiabre.cfm?codlegi=562, acessada em dezembro 2020.

35. http://www2.mma.gov.br/port/conama/legiabre.cfm?codlegi=459, acessada em dezembro 2020.

36. Mojsak, P.; Łozowicka, B.; Kaczyński, P.; Ecotoxicol. Environ. Saf. 2018, 159, 182.

37. Mac Loughlin, T. M.; Peluso, M. L.; Etchegoyen, M. A.; Alonso, L. L.; de Castro, M. C.; Percudani, M. C.; Marino, D. J. G.; Food Control 2018, 93, 129.

38. Bandeira, D. D.; Munaretto, J. S.; Rizzetti, T. M.; Ferronato, G.; Prestes, O. D.; Martins, M. L.; Zanella, R.; Adaime, M. B.; Quim. Nova 2014, 37,900 .

39. http://portal.anvisa.gov.br/programa-de-analise-de-registro-deagrotoxicos-para, acessada em dezembro 2020.

40. https://www.gov.br/agricultura/pt-br/assuntos/inspecao/produtos-animal/ plano-de-nacional-de-controle-de-residuos-e-contaminantes, acessada em dezembro 2020.

41. Britto, F. B.; Do Vasco, A. N.; Pereira, A. P. S.; Méllo, A. V.; Nogueira, L. C.; Rev. Ciênc. Agron. 2012, 43, 390.

42. Wong, H. L.; Garthwaite, D. G.; Ramwell, C. T.; Brown, C. D.; Sci. Total Environ. 2018, 619-620, 874.

43. Damalas, C. A.; Koutroubas, S. D.; Toxics 2016, $4,1$.

44. Lammoglia, S. K.; Kennedy, M. C.; Barriuso, E.; Alletto, L.; Justes, E.; Munier-Jolain, N.; Mamy, L.; Environ. Int. 2017, 105, 66.

45. Polledri, E.; Mercadante, R.; Nijssen, R.; Consonni, D.; Mol, H.; Fustinoni, S.; Sci. Total Environ. 2019, 687, 808.

46. Holt, E.; Audy, O.; Booij, P.; Melymuk, L.; Prokes, R.; Klánová, J.; Sci. Total Environ. 2017, 609, 598.

47. Bolognesi, C.; Merlo, F. D. In Encyclopedia of Environmental Health; Nriagu, J., ed.; $2^{\text {nd }}$ ed., Elsevier: Amsterdam, 2019.

48. Konthonbut, P.; Kongtip, P.; Nankongnab, N.; Tipayamongkholgul, M.; Yoosook, W.; Woskie, S.; Hum. Ecol. Risk Assess. 2019, 0, 1.

49. Abreu, P. H. B. de; Alonzo, H. G. A.; Rev. Bras. Saúde Ocup. 2016, 41, 1.

50. Colosio, C.; Alegakis, A. K.; Tsatsakis, A. M.; Toxicology 2013, 307,1 .

51. Barrón Cuenca, J.; Tirado, N.; Vikström, M.; Lindh, C. H.; Steinus, U.; Leander, K.; Berglund, M.; Dreij, K.; J. Expo. Sci. Environ. Epidemiol. 2019.

52. https://www.inca.gov.br/exposicao-no-trabalho-e-no-ambiente/ agrotoxicos, acessado em dezembro 2020.

53. Han, X.; Zhang, F.; Meng, L.; Xu, Y.; Li, Y.; Li, A.; Turyk, M. E.; Yang, R.; Wang, P.; Zhang, J.; Zhang, Q.; Jiang, G.; Ecotoxicol. Environ. Saf. 2020, 190

54. Czajka, M.; Matysiak-Kucharek, M.; Jodłowska-Jędrych, B.; Sawicki, K.; Fal, B.; Drop, B.; Kruszewski, M.; Kapka-Skrzypczak, L.; Environ. Res. 2019, 178, 108685 .

55. Athina-Maria, A.; Siokas, V.; Sapouni, E.-M.; Sita, N.; Liampas, I.; Brotis, A. G.; Rakitskii, V. N.; Burykina, T. I.; Aschner, M.; Bogdanos, D. P.; Tsatsakis, A.; Hadjigeorgiou, G. M.; Dardiotis, E.; Sci. Total Environ. 2020, 744, 140591

56. Zaganas, I.; Kapetanaki, S.; Mastorodemos, V.; Kanavouras, K.; Colosio, C.; Wilks, M. F.; Tsatsakis, A. M.; Toxicology 2013, 307, 3.

57. Johnson, F. O.; Atchison, W. D.; Neurotoxicology 2009, 30, 761.

58. Weselak, M.; Arbuckle, T. E.; Wigle, D. T.; Walker, M. C.; Krewski, D.; Reprod. Toxicol. 2008, 25, 472.

59. González, J. G.; Miranda, M. I. V.; Mullor, M. R.; Jerez, A. F. H.; Carreño, T. P.; Rodriguez, R. A.; Reprod. Toxicol. 2017, 71, 95.
60. Amaral, A. F. S.; Front. Public Heal. 2014, 2, 716.

61. Georgiadis, N.; Tsarouhas, K.; Tsitsimpikou, C.; Vardavas, A.; Rezaee, R.; Germanakis, I.; Tsatsakis, A.; Stagos, D.; Kouretas, D.; Toxicol. Appl. Pharmacol. 2018, 353, 1.

62. Mostafalou, S.; Abdollahi, M.; Toxicol. Appl. Pharmacol. 2013, 268, 157.

63. Greim, H.; Toxicol. Lett. 2001, 120, 395.

64. Cragin, L. A.; Kesner, J. S.; Bachand, A. M.; Barr, D. B.; Meadows, J. W.; Krieg, E. F.; Reif, J. S.; Environ. Res. 2011, 111, 1293.

65. Paul, K. C.; Sinsheimer, J. S.; Rhodes, S. L.; Cockburn, M.; Bronstein, J.; Ritz, B.; Environ. Health Perspect. 2016, 124, 570.

66. Parrón, T.; Requena, M.; Hernández, A. F.; Alarcón, R.; Toxicol. Appl. Pharmacol. 2011, 256, 379.

67. Freire, C.; Koifman, S.; Int. J. Hyg. Environ. Health 2013, 216, 445.

68. Koh, S. B.; Kim, T. H.; Min, S.; Lee, K.; Kang, D. R.; Choi, J. R.; Neurotoxicology 2017, 62, 181.

69. Park, S.; Kim, S. K.; Kim, J. Y.; Lee, K.; Choi, J. R.; Chang, S. J.; Chung, C. H.; Park, K. S.; Oh, S. S.; Koh, S. B.; Neurotoxicology 2019, 70,12 .

70. Kass, L.; Gomez, A. L.; Altamirano, G. A.; Mol. Cell. Endocrinol. 2020, 508, 110789.

71. Kumar, V.; Yadav, C. S.; Singh, S.; Goel, S.; Ahmed, R. S.; Gupta, S.; Grover, R. K.; Banerjee, B. D.; Chemosphere 2010, 81, 464.

72. Bonner, M. R.; Beane Freeman, L. E.; Hoppin, J. A.; Koutros, S.; Sandler, D. P.; Lynch, C. F.; Hines, C. J.; Thomas, K.; Blair, A.; Alavanja, M. C. R.; Environ. Health Perspect. 2017, 125, 544.

73. Hu, L.; Luo, D.; Zhou, T.; Tao, Y.; Feng, J.; Mei, S.; Environ. Pollut. 2017, 231, 319 .

74. Van Maele-Fabry, G.; Gamet-Payrastre, L.; Lison, D.; Int. J. Hyg. Environ. Health 2019, 222, 49.

75. Sun, F.; Anantharam, V.; Latchoumycandane, C.; Kanthasamy, A.; Kanthasamy, A. G.; J. Pharmacol. Exp. Ther. 2005, 315, 69.

76. Paul, S.; BioEssays 2008, 30, 1172.

77. Back, S. H.; Kang, S.-W.; Han, J.; Chung, H.-T.; Exp. Diabetes Res. 2012, 2012, 1 .

78. Doyle, K. M.; Kennedy, D.; Gorman, A. M.; Gupta, S.; Healy, S. J. M.; Samali, A.; J. Cell. Mol. Med. 2011, 15, 2025.

79. Parrón, T.; Requena, M.; Hernández, A. F.; Alarcón, R.; Toxicol. Lett. 2014, 230, 157

80. Islam, F.; Wang, J.; Farooq, M. A.; Khan, M. S. S.; Xu, L.; Zhu, J.; Zhao, M.; Muños, S.; Li, Q. X.; Zhou, W.; Environ. Int. 2018, 111, 332.

81. Barrón Cuenca, J.; Tirado, N.; Barral, J.; Ali, I.; Levi, M.; Stenius, U.; Berglund, M.; Dreij, K.; Sci. Total Environ. 2019, 695, 133942.

82. Rizzati, V.; Briand, O.; Guillou, H.; Gamet-Payrastre, L.; Chem. Biol. Interact. 2016, 254, 231.

83. Hernández, A. F.; Parrón, T.; Tsatsakis, A. M.; Requena, M.; Alarcón, R.; López-Guarnido, O.; Toxicology 2013, 307, 136.

84. Pundir, C. S.; Malik, A.; Preety; Biosens. Bioelectron. 2019, 140, 111348.

85. Sandoval, L.; Rosca, A.; Oniga, A.; Zambrano, A.; Ramos, J. J.; González, M. C.; Liste, I.; Motas, M.; Sci. Total Environ. 2019, 683, 445.

86. Araújo, C. R. M.; Santos, V. L. A.; Gonsalves, A. A.; Rev. Virtual Quim. 2016, $8,1818$.

87. Cavaliere, M. J.; Calore, E. E.; Perez, N. M.; Rodrigues Puga, F.; Rev. Saude Publica 1996, 30, 267.

88. de Sousa, F. V.; Resende, F. A.; Watanabe, G. A.; da Mata, J. P. ; Cardoso, J. P. G.; Sacramento, L. M.; Gontijo, N. P.; de Moraes, S. M.; Couto, R. C.; Rev. Med. Minas Gerais 2009, 19 (2 Suppl. 3), 50.

89. Suarez-Lopez, J. R.; Hood, N.; Suárez-Torres, J.; Gahagan, S.; Gunnar, M. R.; López-Paredes, D.; Int. J. Hyg. Environ. Health 2019, 222, 981.

90. Samsidar, A.; Siddiquee, S.; Shaarani, S. M.; Trends Food Sci. Technol. 2018, 71, 188. 
91. Yusa, V.; Millet, M.; Coscolla, C.; Roca, M.; Anal. Chim. Acta 2015, $891,15$.

92. Montagner, C. C.; Vidal, C.; Acayaba, R. D.; Quim. Nova 2017, 40, 1094.

93. Skaggs, C. S.; Alluhayb, A. H.; Logue, B. A.; J. Chromatogr. A 2020, 1622, 461102.

94. Mekonen, S.; Argaw, R.; Simanesew, A.; Houbraken, M.; Senaeve, D.; Ambelu, A.; Spanoghe, P.; Chemosphere 2016, 162, 252.

95. Kamel, A.; Byrne, C.; Vigo, C.; Ferrario, J.; Stafford, C.; Verdin, G.; Siegelman, F.; Knizner, S.; Hetrick, J.; Water Res. 2009, 43, 522.

96. Farajzadeh, M. A.; Asghari, A.; Feriduni, B.; J. Food Compos. Anal. 2016, 48, 73 .

97. Ma, Y.; Wen, Y.; Li, J.; Wang, H.; Ding, Y.; Chen, L.; Cent. Eur. J. Chem. 2013, 11, 394

98. Della-Flora, A.; Wielens Becker, R.; Frederigi Benassi, S.; Theodoro Toci, A.; Cordeiro, G. A.; Ibáñez, M.; Portolés, T.; Hernández, F.; Boroski, M.; Sirtori, C.; Sci. Total Environ. 2019, 669, 248.

99. Ccanccapa, A.; Masiá, A.; Navarro-Ortega, A.; Picó, Y.; Barceló, D.; Environ. Pollut. 2016, 211, 414.

100. Machado, K. C.; Grassi, M. T.; Vidal, C.; Pescara, I. C.; Jardim, W. F.; Fernandes, A. N.; Sodré, F. F.; Almeida, F. V.; Santana, J. S.; Canela, M. C.; Nunes, C. R. O.; Bichinho, K. M.; Severo, F. J. R.; Sci. Total Environ. 2016, 572, 138.

101. Montagner, C. C.; Vidal, C.; Acayaba, R. D.; Jardim, W. F.; Jardim, I. C. S. F.; Umbuzeiro, G. A.; Anal. Methods 2014, 6, 6668.

102. Montiel-León, J. M.; Vo Duy, S.; Munoz, G.; Bouchard, M. F.; Amyot, M.; Sauvé, S.; Sci. Total Environ. 2019, 671, 578.

103. Hurtado-Sánchez, M. C.; Romero-González, R. R.; Rodríguez-Cáceres, M. I.; Durán-Merás, I.; Frenich, A. G.; J. Chromatogr. A 2013, 1305, 193.

104. Postigo, C.; López de Alda, M. J.; Barceló, D.; Ginebreda, A.; Garrido, T.; Fraile, J.; J. Hydrol. 2010, 383, 83.

105. Filho, A. M.; dos Santos, F. N.; Pereira, P. A. de P.; Microchem. J. 2010, 96, 139.

106. Jabali, Y.; Millet, M.; El-hoz, M.; Microchem. J. 2019, 147, 83.

107. Bueno, M. J. M.; Gomez, M. J.; Herrera, S.; Hernando, M. D.; Agüera, A.; Fernández-Alba, A. R.; Environ. Pollut. 2012, 164, 267.

108. Pérez-Fernández, V.; Mainero Rocca, L.; Tomai, P.; Fanali, S.; Gentili, A.; Anal. Chim. Acta 2017, 983, 9.

109. de Souza, L. F. C. B.; Montagner, C. C.; Almeida, M. B.; Kuroda, E. K.; Vidal, C.; Freire, R. L.; Semin. Agrar. 2019, 40, 1153.

110. Caldas, S. S.; Arias, J. L. O.; Rombaldi, C.; Mello, L. L.; Cerqueira, M. B. R.; Martins, A. F.; Primel, E. G.; J. Braz. Chem. Soc. 2019, 30, 71.

111. Vashisht, D.; Kumar, A.; Mehta, S. K.; Ibhadon, A.; Environ. Adv. 2020, 1,100002 .

112. Martins, M. L.; Prestes, O. D.; Adaime, M. B.; Zanella, R.; Anal. Methods 2014, 6, 5020.

113. Han, Q.; Wang, Z.; Xia, J.; Xia, L.; Chen, S.; Zhang, X.; Ding, M.; J. Sep. Sci. 2013, 36, 3586.

114. Borrull, J.; Colom, A.; Fabregas, J.; Borrull, F.; Pocurull, E.; J. Chromatogr. A 2020, 1621, 461090.

115. Dong, H.; Xu, L.; Mao, Y.; Wang, Y.; Duan, S.; Lian, J.; Li, J.; Yu, J.; Qiang, Z.; J. Hazard. Mater. 2020, 123986.

116. do Carmo, S. N.; Mendes, L. D.; Corazza, G.; Comelli, H.; Merib, J.; Carasek, E.; Environ. Sci. Pollut. Res. 2020.

117. Elfikrie, N.; Ho, Y. Bin; Zaidon, S. Z.; Juahir, H.; Tan, E. S. S.; Sci. Total Environ. 2020, 712.

118. Liu, S.; Huang, X.; Hu, K.; Jin, Q.; Zhu, G.; J. Chromatogr. Sci. 2020, $58,195$.

119. Almeida, M. B.; Madeira, T. B.; Watanabe, L. S.; Meletti, P. C.; Nixdorf, S. L.; J. Braz. Chem. Soc. 2019, 30, 1657.

120. Corazza, G.; Merib, J.; Do Carmo, S. N.; Mendes, L. D.; Carasek, E.; J. Braz. Chem. Soc. 2019, 30, 1211.
121. Zhang, H.; West, D.; Shi, H.; Ma, Y.; Adams, C.; Eichholz, T.; Water. Air. Soil Pollut. 2019, 230.

122. Solano, M. de L. M.; Montagner, C. C.; Vaccari, C.; Jardim, W. F.; Anselmo-Franci, J. A.; Carolino, R. de O. G.; Luvizutto, J. F.; Umbuzeiro, G. de A.; de Camargo, J. L.; Endocr. Disruptors 2015, 3, e983384.

123. Badach, H.; Nazimek, T.; Kamińska, I. A.; Ann. Agric. Environ. Med. 2007, 14, 109.

124. Kaserzon, S. L.; Heffernan, A. L.; Thompson, K.; Mueller, J. F.; Gomez Ramos, M. J.; Chemosphere 2017, 182, 656.

125. Rubirola, A.; Boleda, M. R.; Galceran, M. T.; J. Chromatogr. A 2017, 1493, 64.

126. Mann, O.; Pock, E.; Wruss, K.; Wruss, W.; Krska, R.; Int. J. Environ. Anal. Chem. 2016, 96, 353

127. Beceiro-González, E.; González-Castro, M. J.; Pouso-Blanco, R.; Muniategui-Lorenzo, S.; López-Mahía, P.; Prada-Rodríguez, D.; Green Chem. Lett. Rev. 2014, 7, 271.

128. Timofeeva, I.; Shishov, A.; Kanashina, D.; Dzema, D.; Bulatov, A.; Talanta 2017, 167, 761.

129. Bedendo, G. C.; Jardim, I. C. S. F.; Carasek, E.; Talanta 2012, 88 , 573.

130. Farajzadeh, M. A.; Feriduni, B.; Afshar Mogaddam, M. R.; Anal. Chim. Acta 2015, 885, 122.

131. Nguyen, T. D.; Lee, M. H.; Lee, G. H.; Microchem. J. 2010, 95, 113.

132. Farina, Y.; Abdullah, M. P.; Bibi, N.; Khalik, W. M. A. W. M.; Food Chem. 2017, 224, 186.

133. Liu, Y.; Gao, Z.; Wu, R.; Wang, Z.; Chen, X.; Chan, T. W. D.; J. Chromatogr. A 2017, 1479, 55.

134. Sivaperumal, P.; Anand, P.; Riddhi, L.; Food Chem. 2015, 168, 356.

135. Li, N.; Chen, J.; Shi, Y. P.; Talanta 2015, 141, 212.

136. Machado, I.; Gérez, N.; Pistón, M.; Heinzen, H.; Cesio, M. V.; Food Chem. 2017, 227, 227.

137. Ribeiro Begnini Konatu, F.; Breitkreitz, M. C.; Sales Fontes Jardim, I. C.; J. Chromatogr. A 2017, 1482, 11.

138. Andrade, G. C. R. M.; Monteiro, S. H.; Francisco, J. G.; Figueiredo, L. A.; Botelho, R. G.; Tornisielo, V. L.; Food Chem. 2015, 175, 57.

139. Han, Y.; Zou, N.; Song, L.; Li, Y.; Qin, Y.; Liu, S.; Li, X.; Pan, C.; J. Chromatogr. B Anal. Technol. Biomed. Life Sci. 2015, 1005, 56.

140. Liu, J.; Tong, L.; Li, D.; Ment, W.; Sun, W.; Zhao, Y.; Yu, Z.; J. Chromatogr. B Anal. Technol. Biomed. Life Sci. 2016, 1017-1018, 233.

141. Esturk, O.; Yakar, Y.; Ayhan, Z.; J. Food Sci. Technol. 2014, 51, 458.

142. Zhang, S.; Yang, Q.; Yang, X.; Wang, W.; Li, Z.; Zhang, L.; Wang, C.; Wang, Z.; Talanta 2017, 166, 46.

143. Saraji, M.; Jafari, M. T.; Mossaddegh, M.; J. Chromatogr. A 2016, 1429 , 30.

144. Pelit, F. O.; Pelit, L.; Dizdaş, T. N.; Aftafa, C.; Ertaş, H.; Yalçinkaya, E. E.; Türkmen, H.; Ertaş, F. N.; Anal. Chim. Acta 2015, 859, 37.

145. Wu, L.; Hu, M.; Li, Z.; Song, Y.; Zhang, H.; Yu, A.; Ma, Q.; Wang, Z.; J. Chromatogr. A 2015, 1407, 42.

146. Tsiropoulos, N. G.; Amvrazi, E. G.; J. AOAC Int. 2011, 94, 634.

147. Wu, L.; Hu, M.; Li, Z.; Song, Y.; Yu, C.; Zhang, H.; Yu, A.; Ma, Q.; Wang, Z.; Food Chem. 2016, 192, 596.

148. Costa, A. I. G.; Queiroz, M. E. L. R.; Neves, A. A.; De Sousa, F. A.; Zambolim, L.; Food Chem. 2015, 181, 64.

149. Nakano, V. E.; Kussumi, T. A.; Lemes, V. R. R.; Kimura, I. de A.; Rocha, S. B.; Alaburda, J.; de Oliveira, M. C. C.; Ribeiro, R. A.; Faria, A. L. R.; Waldhelm, K. C.; Food Sci. Technol. 2016, 36, 40

150. Heleno, F. F.; Rodrigues, A. A. Z.; Queiroz, M. E. L. R.; Neves, A. A.; Oliveira, A. F.; Libardi, V. M.; Microchem. J. 2019, 148, 79.

151. Santana, L. M. B. M.; Gama, A. F.; do Nascimento, R. F.; Cavalcante, R. M.; Accredit. Qual. Assur. 2020, 25, 185.

152. Farajzadeh, M. A.; Afshar Mogaddam, M. R.; J. Chromatogr. A 2016, $1431,8$. 
153. Mahpishanian, S.; Sereshti, H.; Baghdadi, M.; J. Chromatogr. A 2015 , $1406,48$.

154. Farajzadeh, M. A.; Khoshmaram, L.; Nabil, A. A. A.; J. Food Compos. Anal. 2014, 34, 128.

155. Ferreira, V. B.; Estrella, L. F.; Alves, M. G. R.; Gallistl, C.; Vetter, W.; Silva, T. T. C.; Malm, O.; Torres, J. P. M.; Abadio Finco, F. D. B.; J. Environ. Sci. Health, Part B 2020, 55, 30.

156. Araújo, E. A.; Lara, M. C. R.; dos Reis, M. R.; Viriato, R. L. S.; Rocha, R. A. R.; Gonçalves, R. G. L.; Heleno, F. F.; de Queiroz, M. E. L. R.; Tronto, J.; Pinto, F. G.; Food Anal. Methods 2016, 9, 1344.

157. dos Santos, G. M. A. D. A.; Teixeira, L. J. Q.; Pereira, O. S.; dos Santos, A. R.; Fronza, M.; da Silva, A. G.; Scherer, R.; Quim. Nova 2015, 38, 848.

158. Shamsipur, M.; Yazdanfar, N.; Ghambarian, M.; Food Chem. 2016, 204 , 289.

159. Reichert, B.; Pereira Nunes, M. G.; Pizzutti, I. R.; Costabeber, I. H.; Fontana, M. Z.; Jänich, B. D.; Panciera, M. P.; Arbusti, D.; Cardoso, C. D.; Chim, J. F.; J. Sci. Food Agric. 2020, 100, 2425.

160. Li, Y. F.; Qiao, L. Q.; Li, F. W.; Ding, Y.; Yang, Z. J.; Wang, M. L.; J. Chromatogr. A 2014, 1361, 77.

161. Yang, X.; Qiao, K.; Liu, F.; Wu, X.; Yang, M.; Li, J.; Gao, H.; Zhang, S.; Zhou, W.; Lu, R.; Talanta 2017, 166, 93.

162. Abd-Alrahman, S. H.; Food Chem. 2014, 159, 1.

163. Topuz, S.; Özhan, G.; Alpertunga, B.; Food Control 2005, 16, 87.

164. Silva, R.; Faria, A.; Quim. Nova 2020, X, 1.

165. Silva, H. C. M. P. da; Bedor, D. C. G.; Cunha, A. N.; Rodrigues, H. O. dos S.; Telles, D. L.; Araújo, A. C. P.; Santana, D. P.; Food Addit. Contam., Part B 2020, 13, 16.

166. Souza, D. F.; Souza, E. L.; Borges, E. M.; J. Braz. Chem. Soc. 2016, 27 , 1626.

167. Kemmerich, M.; Rizzetti, T. M.; Martins, M. L.; Prestes, O. D.; Adaime, M. B.; Zanella, R.; Food Anal. Methods 2015, 8, 728.

168. Lemos, M. F.; Lemos, M. F.; Pacheco, H. P.; Scherer, R.; Quim. Nova 2015, 38, 268

169. Denghel, H.; Göen, T.; Toxicol. Lett. 2018, 298, 33.

170. Haines, D. A.; Saravanabhavan, G.; Werry, K.; Khoury, C.; Int. J. Hyg. Environ. Health 2017, 220, 13.

171. Needham, L. L.; Calafat, A. M.; Barr, D. B.; Int. J. Hyg. Environ. Health 2007, 210, 229.

172. Barr, D. B.; J. Chem. Health Saf. 2008, 15, 20.

173. Esteban, M.; Castaño, A.; Environ. Int. 2009, 35, 438.

174. Kapka-Skrzypczak, L.; Cyranka, M.; Skrzypczak, M.; Kruszewski, M.; Ann. Agric. Environ. Med. 2011, 18, 294.

175. Connolly, A.; Jones, K.; Basinas, I.; Galea, K. S.; Kenny, L.; McGowan, P.; Coggins, M. A.; Int. J. Hyg. Environ. Health 2019, 222, 205.

176. Yusa, V.; Millet, M.; Coscolla, C.; Pardo, O.; Roca, M.; Chemosphere 2015, 139, 91 .

177. Tao, Y.; Phung, D.; Dong, F.; Xu, J.; Liu, X.; Wu, X.; Liu, Q.; He, M.; Pan, X.; Li, R.; Zheng, Y.; Sci. Total Environ. 2019, 669, 721.

178. Panuwet, P.; Hunter, R. E.; D’Souza, P. E.; Chen, X.; Radford, S. A.; Cohen, J. R.; Marder, M. E.; Kartavenka, K.; Ryan, P. B.; Barr, D. B.; Crit. Rev. Anal. Chem. 2016, 46, 93.

179. Duca, R. C.; Salquebre, G.; Hardy, E.; Appenzeller, B. M. R.; J. Chromatogr. B 2014, 955-956, 98.

180. Vogliardi, S.; Tucci, M.; Stocchero, G.; Ferrara, S. D.; Favretto, D.; Anal. Chim. Acta 2015, 857, 1.

181. Mannetje, A. t.; Coakley, J.; Mueller, J. F.; Harden, F.; Toms, L. M.; Douwes, J.; Chemosphere 2012, 89, 911.

182. Du, J.; Gridneva, Z.; Gay, M. C. L.; Lai, C. T.; Trengove, R. D.; Hartmann, P. E.; Geddes, D. T.; Sci. Rep. 2016, 6, 1.

183. Jeong, Y.; Lee, S.; Kim, S.; Choi, S. D.; Park, J.; Kim, H. J.; Lee, J. J.; Choi, G.; Choi, S.; Kim, S.; Kim, S. Y.; Kim, Y. D.; Cho, G.; Suh, E.; Kim, S. K.; Eun, S. H.; Eom, S.; Kim, S.; Kim, G. H.; Kim, S.; Choi, K.; Moon, H. B.; Environ. Res. 2016, 147, 8.
184. Ostrea, E. M.; Bielawski, D. M.; Posecion, N. C.; Corrion, M.; Villanueva-Uy, E.; Jin, Y.; Janisse, J. J.; Ager, J. W.; Environ. Res. 2008, 106, 277.

185. Tsatsakis, A. M.; Barbounis, M. G.; Kavalakis, M.; Kokkinakis, M.; Terzi, I.; Tzatzarakis, M. N.; J. Chromatogr. B 2010, 878, 1246.

186. Berton, T.; Mayhoub, F.; Chardon, K.; Duca, R. C.; Lestremau, F.; Bach, V.; Tack, K.; Environ. Res. 2014, 132, 311.

187. Hernández, A. F.; Lozano-Paniagua, D.; González-Alzaga, B.; Kavvalakis, M. P.; Tzatzarakis, M. N.; López-Flores, I.; AguilarGarduño, C.; Caparros-Gonzalez, R. A.; Tsatsakis, A. M.; Lacasaña, M.; Environ. Int. 2019, 131, 104997.

188. Hardy, E. M.; Duca, R. C.; Salquebre, G.; Appenzeller, B. M. R.; Forensic Sci. Int. 2015, 249, 6.

189. Batterman, S.; Chernyak, S.; Sci. Total Environ. 2014, 494-495, 252.

190. Çok, I.; Mazmanci, B.; Mazmanci, M. A.; Turgut, C.; Henkelmann, B.; Schramm, K. W.; Environ. Int. 2012, 40, 63.

191. Chen, X.; Panuwet, P.; Hunter, R. E.; Riederer, A. M.; Bernoudy, G. C.; Barr, D. B.; Ryan, P. B.; J. Chromatogr. B Anal. Technol. Biomed. Life Sci. 2014, 970, 121.

192. Meyer-Monath, M.; Chatellier, C.; Cabooter, D.; Rouget, F.; Morel, I.; Lestremau, F.; Talanta 2015, 138, 231.

193. Chen, D.; Miao, H.; Zhao, Y.; Wu, Y.; J. Chromatogr. A 2019, 1587, 73.

194. Cazorla-Reyes, R.; Fernández-Moreno, J. L.; Romero-González, R.; Frenich, A. G.; Vidal, J. L. M.; Talanta 2011, 85, 183.

195. Sweeney, C.; Park, Y.; Kim, J. S.; J. Chromatogr. A 2019, 1603, 83.

196. Garí, M.; González-Quinteiro, Y.; Bravo, N.; Grimalt, J. O.; Sci. Total Environ. 2018, 622-623, 526.

197. Liao, H. T.; Hsieh, C. J.; Chiang, S. Y.; Lin, M. H.; Chen, P. C.; Wu, K. Y.; J. Chromatogr. B 2011, 879, 1961.

198. López-García, M.; Romero-González, R.; Garrido Frenich, A.; J. Pharm. Biomed. Anal. 2019, 173, 31.

199. López-García, M.; Romero-González, R.; Lacasaña, M.; Garrido Frenich, A.; J. Pharm. Biomed. Anal. 2017, 146, 378.

200. Wittsiepe, J.; Nestola, M.; Kohne, M.; Zinn, P.; Wilhelm, M.; J. Chromatogr. B 2014, 945-946, 217.

201. Koivunen, M. E.; Dettmer, K.; Vermeulen, R.; Bakke, B.; Gee, S. J.; Hammock, B. D.; Anal. Chim. Acta 2006, 572, 180.

202. Salquèbre, G.; Schummer, C.; Millet, M.; Briand, O.; Appenzeller, B. M. R.; Anal. Chim. Acta 2012, 710, 65 .

203. Peng, F. J.; Hardy, E. M.; Mezzache, S.; Bourokba, N.; Palazzi, P.; Stojiljkovic, N.; Bastien, P.; Li, J.; Soeur, J.; Appenzeller, B. M. R.; Environ. Int. 2020, 138, 105633.

204. Flores-Ramírez, R.; Ortiz-Pérez, M. D.; Batres-Esquivel, L.; Castillo, C. G.; Ilizaliturri-Hernández, C. A.; Díaz-Barriga, F.; Talanta 2014, 123, 169.

205. Kim, M.; Song, N. R.; Hong, J.; Lee, J.; Pyo, H.; Chemosphere 2013, 92, 279.

206. Béranger, R.; Hardy, E. M.; Dexet, C.; Guldner, L.; Zaros, C.; Nougadère, A.; Metten, M. A.; Chevrier, C.; Appenzeller, B. M. R.; Environ. Int. 2018, 120, 43.

207. Lehmann, E.; Oltramare, C.; de Alencastro, L. F.; Anal. Chim. Acta 2018, 999, 87.

208. Iqbal, S.; Iqbal, M. M.; Javed, M.; Bahadur, A.; Yasien, S.; Najam-uddin; Hurr, A.; Ahmad, N.; Raheel, M.; Liu, G.; J. Chromatogr. B 2020, $1152,122227$.

209. Lehmann, E.; Oltramare, C.; Nfon Dibié, J. J.; Konaté, Y.; de Alencastro, L. F.; Environ. Int. 2018, 111, 317.

210. Roca, M.; Leon, N.; Pastor, A.; Yusà, V.; J. Chromatogr. A 2014, 1374, 66.

211. Luzardo, O. P.; Ruiz-Suárez, N.; Almeida-González, M.; HenríquezHernández, L. A.; Zumbado, M.; Boada, L. D.; Anal. Bioanal. Chem. 2013, 405, 9523. 
212. Luzardo, O. P.; Almeida-González, M.; Ruiz-Suárez, N.; Zumbado, M.; Henríquez-Hernández, L. A.; Meilán, M. J.; Camacho, M.; Boada, L. D.; Sci. Justice 2015, 55, 307.

213. Park, M. J.; In, S. W.; Lee, S. K.; Choi, W. K.; Park, Y. S.; Chung, H. S.; Forensic Sci. Int. 2009, 184, 28.

214. Ferrari Júnior, E.; Caldas, E. D.; Forensic Sci. Int. 2018, 290, 318.

215. Guo, H.; Zhang, P.; Wang, J.; Zheng, J.; J. Chromatogr. B 2014, 951 $952,89$.

216. Salihovic, S.; Mattioli, L.; Lindström, G.; Lind, L.; Monica Lind, P.; van Bavel, B.; Chemosphere 2012, 86, 747.

217. Yuan, H.; Jin, J.; Bai, Y.; Li, Q.; Wang, Y.; Wang, Q.; Sci. Total Environ. 2017, 580, 1027.

218. Mercadante, R.; Polledri, E.; Moretto, A.; Fustinoni, S.; Toxicol. Lett. 2018, 298, 19 .
219. Ostrea, E. M.; Morales, V.; Ngoumgna, E.; Prescilla, R.; Tan, E.; Hernandez, E.; Ramirez, G. B.; Cifra, H. L.; Manlapaz, M. L.; Neurotoxicology 2002, 23, 329.

220. Haraux, E.; Tourneux, P.; Kouakam, C.; Stephan-Blanchard, E.; Boudailliez, B.; Leke, A.; Klein, C.; Chardon, K.; Environ. Int. 2018, $119,20$.

221. Lee, S.; Kim, S.; Lee, H. K.; Lee, I. S.; Park, J.; Kim, H. J.; Lee, J. J.; Choi, G.; Choi, S.; Kim, S.; Kim, S. Y.; Choi, K.; Kim, S.; Moon, H. B.; Chemosphere 2013, 93, 1578.

222. Rojas-Squella, X.; Santos, L.; Baumann, W.; Landaeta, D.; Jaimes, A.; Correa, J. C.; Sarmiento, O. L.; Ramos-Bonilla, J. P.; Chemosphere 2013, 91, 733 . 